\title{
VLT/X-shooter spectroscopy of the GRB 120327A afterglow ${ }^{\star}, \star \star$
}

\author{
V. D’Elia ${ }^{1,2}$, J. P. U. Fynbo ${ }^{3}$, P. Goldoni ${ }^{4}$, S. Covino ${ }^{5}$, A. de Ugarte Postigo ${ }^{6,3}$, C. Ledoux ${ }^{7}$, F. Calura ${ }^{8}$, \\ J. Gorosabel ${ }^{6,9,10}$, D. Malesani ${ }^{3}$, F. Matteucci ${ }^{11}$, R. Sánchez-Ramírez ${ }^{6}$, S. Savaglio ${ }^{12,19}$, A. J. Castro-Tirado ${ }^{6}$, \\ O. E. Hartoog ${ }^{13}$, L. Kaper ${ }^{13}$, T. Muñoz-Darias ${ }^{14,15}$, E. Pian $^{16}$, S. Piranomonte ${ }^{1}$, G. Tagliaferri ${ }^{5}$, N. Tanvir ${ }^{17}$, \\ S. D. Vergani ${ }^{18}$, D. J. Watson ${ }^{3}$, and D. $\mathrm{Xu}^{3}$ \\ 1 INAF - Osservatorio Astronomico di Roma, via Frascati 33, 00040 Monteporzio Catone, Italy \\ e-mail: delia@mporzio.astro.it \\ 2 ASI-Science Data Center, via del Politecnico snc, 00133 Rome, Italy \\ 3 Dark Cosmology Centre, Niels Bohr Institute, University of Copenhagen, Juliane Maries Vej 30, 2100 Copenhagen, Denmark \\ 4 APC, Astroparticule et Cosmologie, Univ. Paris Diderot, CNRS/IN2P3, CEA/Irfu, Obs. de Paris, Sorbonne Paris Cité, \\ 10 rue Alice Domon et Léonie Duquet, 75205 Paris Cedex 13, France \\ 5 INAF-Osservatorio Astronomico di Brera, via E. Bianchi 46, 23807 Merate (LC), Italy \\ ${ }^{6}$ Instituto de Astrofísica de Andalucía (IAA-CSIC), Glorieta de la Astronomía s/n, 18008 Granada, Spain \\ 7 European Southern Observatory, Alonso de Cordova 3107, Vitacura, Casilla 19001 Santiago 19, Chile \\ 8 INAF, Osservatorio Astronomico di Bologna, via Ranzani 1, 40127 Bologna, Italy \\ 9 Unidad Asociada Grupo Ciencia Planetarias UPV/EHU-IAA/CSIC, Departamento de Física Aplicada I, E.T.S. Ingenieria, \\ Universidad del Pais Vasco UPV/EHU, Alameda de Urquijo s/n, 48013 Bilbao, Spain \\ ${ }_{10}$ Ikerbasque, Basque Foundation for Science, Alameda de Urquijo 36-5, 48008 Bilbao, Spain \\ 11 INAF, Osservatorio Astronomico di Trieste, via Tiepolo 11, 34143 Trieste, Italy \\ 12 Max Planck Institute for Extraterrestrial Physics, 85748 Garching bei München, Germany \\ 13 Astronomical Institute "Anton Pannekoek", University of Amsterdam, Science Park 904, 1098 XH Amsterdam, The Netherlands \\ 14 School of Physics and Astronomy University of Southampton, Southampton, Hampshire, SO17 1BJ, United Kingdom \\ 15 University of Oxford, Department of Physics, Astrophysics, Keble Road, Oxford, OX1 3RH, UK \\ 16 Scuola Normale Superiore, Piazza dei Cavalieri 7, 56126 Pisa, Italy \\ 17 Department of Physics and Astronomy, University of Leicester, Leicester LE1 7RH, UK \\ 18 GEPI, Observatoire de Paris, CNRS, Univ. Paris Diderot, 5 place Jules Janssen, 92190 Meudon, France \\ 19 Physics Department, University of Calabria, 87036 Rende, Italy
}

Received 15 November 2013 / Accepted 17 February 2014

\section{ABSTRACT}

\begin{abstract}
Aims. We present a study of the environment of the Swift long gamma-ray burst GRB 120327A at $z \approx 2.8$ through optical spectroscopy of its afterglow.

Methods. We analyzed medium-resolution, multi-epoch spectroscopic observations $(R \sim 7000-12000$, corresponding to $\sim 15-23 \mathrm{~km} \mathrm{~s}^{-1}, S / N=15-30$ and wavelength range 3000-25000 $)$ of the optical afterglow of GRB 120327A, taken with X-shooter at the VLT 2.13 and $27.65 \mathrm{hr}$ after the GRB trigger.

Results. The first epoch spectrum shows that the ISM in the GRB host galaxy at $z=2.8145$ is extremely rich in absorption features, with three components contributing to the line profiles. The hydrogen column density associated with GRB 120327A has $\log N_{\mathrm{H}} / \mathrm{cm}^{-2}=22.01 \pm 0.09$, and the metallicity of the host galaxy is in the range $[X / H]=-1.3$ to -1.1 . In addition to the ground state lines, we detect absorption features associated with excited states of C II, O I, Si II, Fe II, and Ni II, which we used to derive information on the distance between the host absorbing gas and the site of the GRB explosion. The variability of the Fe II $\lambda 2396$ excited line between the two epochs proves that these features are excited by the GRB UV flux. Moreover, the distance of component I is found to be $d_{\mathrm{I}}=200_{-60}^{+100} \mathrm{pc}$, while component II is located closer to the GRB, at $d_{\mathrm{II}}=100_{-30}^{+40} \mathrm{pc}$. These values are among the lowest found in GRBs. Component III does not show excited transitions, so it should be located farther away from the GRB. The presence of $\mathrm{H}_{2}$ molecules is firmly established, with a molecular fraction $f$ in the range $f=4 \times 10^{-7}-10^{-4}$. This particularly low value can be attributed to the small dust content. This represents the third positive detection of molecules in a GRB environment.
\end{abstract}

Key words. ISM: abundances - dust, extinction - ISM: molecules - galaxies: ISM

\section{Introduction}

For a few hours after their onset, long gamma-ray burst (GRB) afterglows (see, e.g., Mészáros 2006 for a review) are the

\footnotetext{
* Based on observations collected at the European Southern Observatory, ESO, the VLT/Kueyen telescope, Paranal, Chile, proposal code: 088.A-0051.

$\star \star$ The reduced spectrum is only available at the CDS via anonymous ftp to cdsarc.u-strasbg. fr $(130.79 .128 .5)$ or via http://cdsarc.u-strasbg.fr/viz-bin/qcat?]/A+A/564/A38
}

brightest sources in the Universe. Their intense and featureless continua in the X-ray to the radio band offer a superb opportunity to study the gas and dust content of high redshift galaxies (see e.g., Vreeswijk et al. 2001, 2014; Berger et al. 2006; Fynbo et al. 2006; Prochaska et al. 2007; Sparre et al. 2013). Therefore, GRBs provide a complementary diagnostic tool to study high-redshift environments, with respect to Lyman-break galaxies (LBGs, see e.g. Steidel et al. 1999), $K$-band selected galaxies (Savaglio et al. 2004; Martin et al. 2012; Kornei et al. 2013), and galaxies that happen to be along the lines of sight 
to bright background quasars (QSOs). In fact, LBG studies are biased towards high UV-luminosity galaxies, and their inferred chemical abundances may not be related to that of typical highredshift galaxies.

Ultra-deep spectroscopic observations may allow us to study normal galaxies, but only up to $z \sim 2$ (using the present generation of 8-m class telescopes). For example, Savaglio et al. (2004, 2005) studied the interstellar medium (ISM) of a sample of faint $K$-band selected galaxies, finding abundances much higher than in QSOs systems. This is because the line of sight to QSO probes mainly galaxy halos, rather than their bulges or discs (Fynbo et al. 2008), returning lower abundance values. GRBs represent a way to study the central regions of high-redshift galaxies, without luminosity biases. Abundances measured in GRB host galaxies vary from less than $10^{-2}$ to solar values and are on average higher than those found along QSO sightlines (see e.g., Fynbo et al. 2006; Prochaska et al. 2007; Savaglio et al. 2012; Sparre et al. 2013). GRBs are expected to originate in molecular clouds, where star formation occurs. Nevertheless, absorption from ground-state and vibrationally excited levels of the $\mathrm{H}_{2}$ and other molecules is hardly observed in GRB afterglow spectra (Vreeswijk et al. 2004; Tumlinson et al. 2007), with the exception of GRB 080607 (Prochaska et al. 2009; Sheffer et al. 2009), GRB 120815A (Krühler et al. 2013), and possibly GRB 060206 (Fynbo et al. 2006). The nondetection of molecules is possibly a consequence of photo-dissociation by the intense UV flux from the GRB afterglow or by the young, hot stellar populations harboring the GRB host.

Furthermore, GRBs are the most variable and violent phenomena in the Universe, while QSOs are persistent, albeit variable, sources. Thus, the physical, dynamical and chemical status of the circumburst medium in the star-forming region hosting GRB progenitors is altered in real time by the explosive event, through shock waves and ionizing photons. The transient nature of GRBs is manifested through variability in the populations of fine structure and other excited levels of the $\mathrm{O} I$ atom and of ions such as Fe II, Ni II, Si II and C II. The population of these excited levels, and therefore the observed column densities of the corresponding absorption lines, change on timescales of minutes to days (Vreeswijk et al. 2007; D'Elia et al. 2009a, 2012; Hartoog et al. 2013). This variation is not consistent with a pure infrared excitation or collisional processes, but is due to UV-pumping (Prochaska et al. 2006; Vreeswijk et al. 2007). Monitoring these variations and comparing them with predictions from photoexcitation codes enables one to derive information on the distance between the GRB and the absorbing material. This distance can be estimated even when multi-epoch spectroscopy is not available, under the assumption that UV pumping is the responsible mechanism for the production of the excited lines (D'Elia et al. 2009b, 2010, 2011; Ledoux et al. 2009).

The GRB environments are in general very complex. In fact, they are constituted by several layers of gas which may be close to or far from one another, have different relative velocities and physical properties. Optical spectroscopy reflects this complexity, since the absorption lines in several cases cannot be fitted with a single line profile. The ISM complexity is particularly evident for GRB afterglows observed at high resolution (Fiore et al. 2005; D'Elia et al. 2007; Thöne et al. 2008; Piranomonte et al. 2008).

Here we present the case of GRB 120327 A, observed by X-shooter on 2012 March 27 and 28. Our dataset is complemented by a GTC spectrum and photometric data from several facilities. We derive the abundances by comparing the column densities of hydrogen and metals, and evaluate the
GRB-absorber distance using the variation of the excited levels and their ratios to the ground state ones, and the predictions of photo-excitation codes. We also characterize the host galaxy using the dust depletion pattern technique and chemical evolution models, derive the extinction curve shape and search for $\mathrm{H}_{2}$ molecules in the GRB environment.

The paper is organized as follows. Section 2 summarizes the properties of GRB 120327A and results of observations previously reported in the literature; Sect. 3 presents our observations and data reduction; Sect. 4 is devoted to the analysis of the absorption features from the host galaxy, using X-shooter data; Sect. 5 illustrates our main results, which are discussed in Sect. 6. Finally, in Sect. 7 the conclusions are drawn. We assume a cosmology with $H_{0}=70 \mathrm{~km} \mathrm{~s}^{-1} \mathrm{Mpc}^{-1}, \Omega_{\mathrm{m}}=0.3$, $\Omega_{\Lambda}=0.7$. Hereafter, the notation $[X / H]$ indicates the logarithmic abundance of element $X$ relative to solar values.

\section{GRB 120327A}

GRB 120327A was discovered by the Burst Alert Telescope (BAT) instrument on board Swift (Gehrels et al. 2004) on 2012 March 27 at 02:55:16 UT (Sbarufatti et al. 2012). The BAT light curve shows 3 main spikes, peaking at $T_{0}+2 \mathrm{~s}, T_{0}+18 \mathrm{~s}$ and $T_{0}+37 \mathrm{~s}$, respectively, where $T_{0}$ is the burst detection time. The last one is the main peak, and the duration for this burst in the $15-350 \mathrm{keV}$ range is $T_{90}=62.9 \pm 7.5 \mathrm{~s}$. The BAT spectrum is best fit by a simple power-law model, with a photon index of $1.52 \pm 0.06$ (Krimm et al. 2012).

The X-Ray Telescope (XRT) and Ultraviolet and the Optical Telescope (UVOT) were repointed toward the GRB position $76 \mathrm{~s}$ and $140 \mathrm{~s}$ after the trigger, respectively. Both instruments detected the afterglow. The XRT light curve shows a typical steep-flat-normal behavior with temporal indices of $\alpha_{1}=2.95$, $\alpha_{2}=0.62$, and $\alpha_{3}=1.42$, with temporal breaks at $217 \mathrm{~s}$ and $2540 \mathrm{~s}$ after the burst (Stroh et al. 2012). The X-ray spectrum can be modeled with an absorbed power law with a spectral index of $\sim 1$ and a column density of $N_{\mathrm{H}} \approx 1.5 \times 10^{22} \mathrm{~cm}^{-2}$, taking the absorption by our Galaxy into account $\left(N_{\mathrm{H}, \mathrm{G}}=1.4 \times 10^{21} \mathrm{~cm}^{-2}\right.$; Kalberla et al. 2005).

Ground-based facilities were pointed to the GRB starting a few minutes later, allowing the detection of the afterglow in the optical and near infrared (NIR; Klotz et al. 2012; Smith \& Virgili 2012; LaCluyze et al. 2012; Covino et al. 2012), and the measurement of a photometric redshift of $z \sim 3$ (Sudilowsky et al. 2012). Optical spectroscopy followed later on, yielding a redshift of 2.813 (Perley \& Tanvir 2012; Krühler et al. 2012; Sánchez-Ramírez et al. 2012). Finally, the afterglow was also detected in the radio band at $34 \mathrm{GHz}, \approx 4.6$ days after the trigger (Hancock et al.2012).

\section{Observations and data reduction}

\subsection{X-shooter observations}

We observed the afterglow of GRB 120327A twice with X-shooter (D'Odorico et al. 2006; de Ugarte Postigo et al. 2010; Vernet et al. 2011), a single-object medium resolution ( $R=\lambda / \Delta \lambda=4000-14000)$ echelle spectrograph mounted at the VLT-UT2 telescope. The observations were carried out under the GTO program 088.A-0051.

For both observations the slit width was set to $0.9^{\prime \prime}$ in the visual (VIS) and near-infrared (NIR) arms, and 1.0" in the ultraviolet and blue (UVB) arm. The UVB and VIS CCD detectors were rebinned to $1 \times 2$ pixels (binned in the spectral direction 
Table 1. X-shooter observations

\begin{tabular}{lccc}
\hline \hline Observation time (UT) & Hours since GRB & Exp. (s) & $S / N$ \\
\hline March 27, 05:03:56 (A) & 2.13 & 600 & $5-15$ \\
March 27, 05:15:51 (B) & 2.33 & 600 & $5-15$ \\
March 27, 05:27:35 (C) & 2.53 & 600 & $5-15$ \\
March 27, 05:39:16 (D) & 2.72 & 600 & $5-15$ \\
A+B+C+D = 1st epoch & 2.47 & 2400 & $10-30$ \\
March 28, 08:42:36 & 27.65 & $\sim 4200$ & $2-5$ \\
\hline
\end{tabular}

but not in the spatial one) to reduce the readout noise. With this configuration, the nominal resolution is different for the three arms: $R \sim 5100,8800,5300$ for the UVB, VIS, and NIR arms, respectively. The first observation began on 2012 March 27 at 05:03:56 UT, $\approx 2.13 \mathrm{hr}$ after the GRB trigger (the magnitude of the afterglow at the time of this observation was $R=18.84 \pm 0.09$, see Klotz et al. 2012). It consisted of 4 different exposures of $600 \mathrm{~s}$ each for a total net exposure of $40 \mathrm{~min}$ (see Table 1). The exposures were taken nodding along the slit with an offset of $5^{\prime \prime}$ between exposures, following a standard ABBA pattern. We started the second sequence the following night, on 2012 March 28, at 08:42:36 UT, using the same instrument configuration. Because of the lower flux, the exposure time of every frame was $1200 \mathrm{~s}$, though the fourth exposure was stopped after $\sim 600 \mathrm{~s}$ because of the incoming twilight.

We processed the spectra using version 1.4 .5 of the $\mathrm{X}$-shooter data reduction pipeline (Goldoni et al. 2006; Modigliani et al. 2010). The pipeline carries out the following steps: the raw frames were first bias-subtracted, and cosmic ray hits were detected and removed using the method developed by van Dokkum (2001). The frames were divided by a master flat field obtained using day-time flat field exposures with halogen lamps. The orders were extracted and rectified in wavelength space using a wavelength solution obtained from day-time calibration frames. The resulting rectified orders were shifted and co-added to obtain the final two-dimensional spectrum. In the overlapping regions, orders were merged by weighing them using the errors propagated during the reduction process. From the resulting two-dimensional merged spectrum, a one-dimensional spectrum with the corresponding error file and bad pixel map was extracted at the source position.

To perform flux calibration we extracted a spectrum from a staring observation of the flux standard star GD 153 (Bohlin $\&$ Gilliland 2004). In this case we subtracted the sky emission lines using the method developed by Kelson (2003). This spectrum was divided by the flux table of the same star from the CALSPEC HST database ${ }^{1}$ to produce the response function. The response was then interpolated where needed inside the atmospheric absorption bands in VIS and NIR and applied to the spectrum of the source. No telluric correction was applied, except while checking for variability of some Fe II lines (see Sect. 4.7).

At a first glance the flux-calibrated VIS spectrum of the first observation appeared to be anomalous with respect to the UVB and NIR portions. Indeed in the overlapping region between the VIS and NIR arm, the flux level of the VIS spectrum was about 10 times lower than the flux level of the NIR spectrum. Moreover the VIS spectrum could be fitted with a power law $F_{\lambda} \propto \lambda^{-3.3}$, inconsistent with the UVB and NIR slopes. No such anomaly is present in the much weaker spectra of the second observation.

\footnotetext{
1 http://www.stsci.edu/hst/observatory/cdbs/calspec. html
}

As our flux calibration was carried out in a standard way, we suspected a technical anomaly to be responsible for this effect. After consulting with the X-shooter instrument scientist at Paranal, Christophe Martayan, we concluded that this anomalous spectrum is the result of a malfunction of the atmospheric dispersion corrector (ADC) of the VIS arm. Indeed, starting from 2012 August, the X-shooter ADCs have been disabled because of recurring problems of this kind. We checked that this phenomenon does not impact the quality of the wavelength calibration but only the flux level. In the following we do not use this flux calibrated VIS spectrum, but only the normalized one.

\subsection{GTC spectroscopic observations}

We also obtained long-slit spectroscopy of the GRB 120327A afterglow with the OSIRIS spectrograph (Cepa et al. 2003) mounted on the 10.4-m Gran Telescopio de Canarias (GTC), located at the Roque de los Muchachos Observatory (SánchezRamírez et al. 2012). We took $3 \times 400 \mathrm{~s}$ spectra with the R1000B grism, covering the range $3626-7883 \AA, 2.33 \mathrm{~h}$ after the BAT trigger. The resolution of our spectra is $R \sim 750$.

The data were reduced using standard procedures in IRAF and the flux calibration is based on the spectrophotometric standard G191-B2B (Oke 1990). We have also scaled the combined spectrum to the magnitude of the acquisition image $\left(r^{\prime}=18.5 \pm\right.$ 0.1 in the $\mathrm{AB}$ system, not corrected for Galactic extinction of $E_{B-V}=0.34 \mathrm{mag}$ ) to compensate for slit losses.

\subsection{Photometric observations}

Late $r^{\prime}$-band images were taken with a total exposure time of $8 \times 600 \mathrm{~s}$ with the 2.0-m LT telescope, between 2013 April 23.15 and 23.21 UT. The observations failed to detect any source above a $3 \sigma$ limit of $r>24.0$ (AB system, corrected for Galactic extinction, and calibrated using the GTC data, see below) coincident with the optical afterglow position (Kuin et al. 2012; Smith \& Virgili 2012). Further $r^{\prime}$-band imaging was carried out with the GTC equipped with OSIRIS. Twelve images with an exposure time of $50 \mathrm{~s}$ per frame were taken on 2012 May 26.096-26.11 UT. The field was calibrated with standard star PG 1047 (Smith et al. 2002) observed at an airmass similar to the one of the GRB field. The combined image reveals an object coincident with the afterglow position (Fig. 1), which we propose to be the host galaxy, since the extrapolation of the afterglow light curve at two months after the GRB explosion results in negligible flux, and no contribution is expected in the rest-frame UV from any underlying supernova. Aperture photometry yields an $\mathrm{AB}$ magnitude of $r^{\prime}=24.50 \pm 0.23$ for the host, including calibration errors and not corrected for Galactic reddening.

Additional late $H$-band imaging was performed with the $3.5 \mathrm{~m}$ Calar Alto telescope equipped with $\Omega_{2000}$. The observations were done on 2012 May 1.0622-1.0902 UT, with a total exposure time of $1800 \mathrm{~s}$. The combined image reveals an object coincident with the GTC detection, with $H=20.09 \pm 0.32$ (Vega) not corrected for Galactic reddening and including the calibration errors. The $H$-band photometric calibration was carried out with 468 field stars of the 2MASS catalog.

In addition, the afterglow $I$-band decay was monitored by means of 14 images of $300 \mathrm{~s}$ each acquired with the IAC80 telescope from 1.19 to $2.63 \mathrm{~h}$ after the GRB (Gorosabel et al. 2012, see also Appendix A). The afterglow shows a smooth fading from $I=17.32 \pm 0.05$ to $I=18.17 \pm 0.13$ (Vega). The $I$-band 

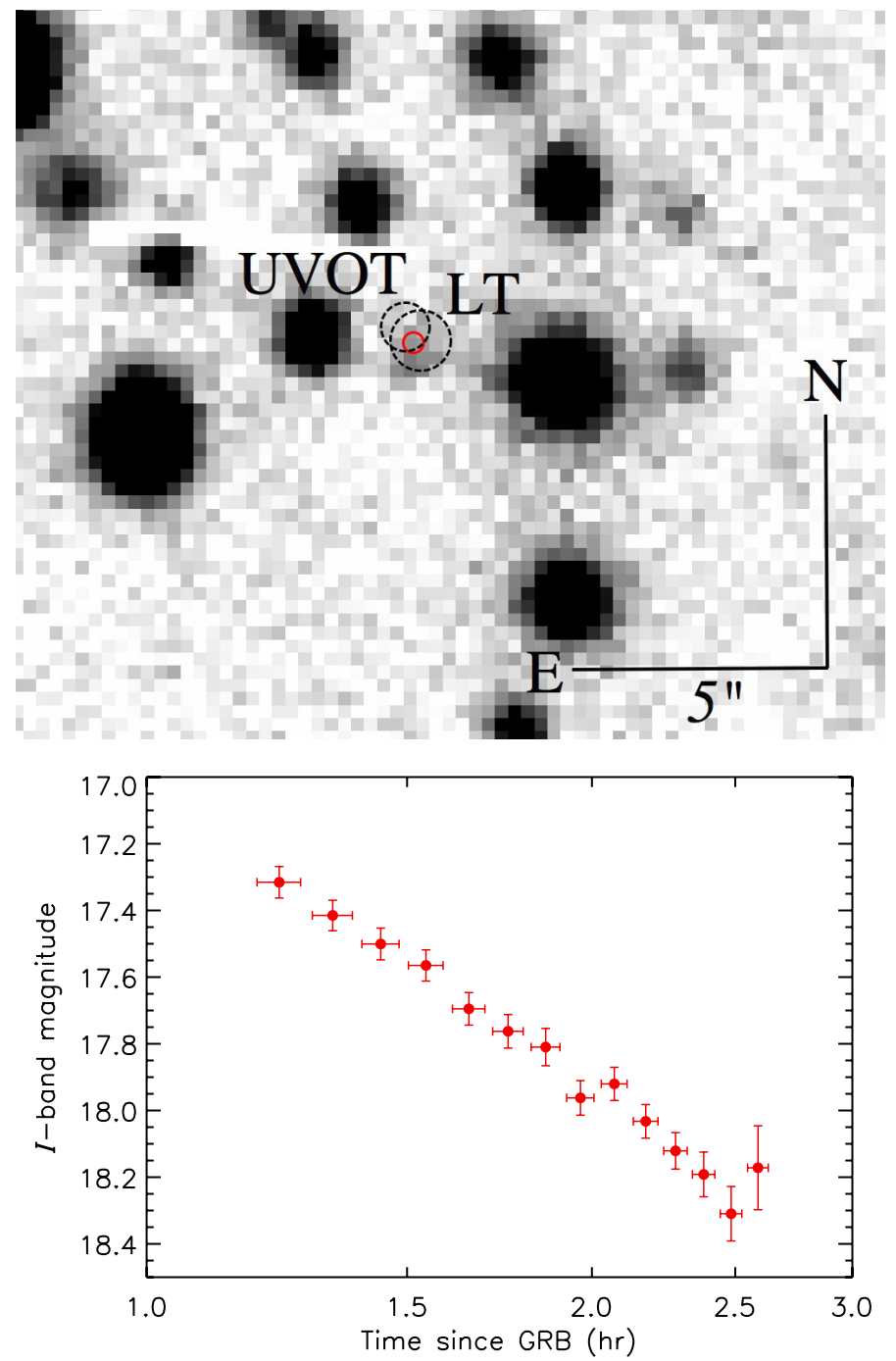

Fig. 1. Upper panel: combined $r^{\prime}$-band image result of the GTC late observations. The two long-dashed circles indicate the astrometric error circles of the UVOT (Kuin \& Sbarufatti 2012) and Liverpool Telescope (Smith \& Virgili 2012) positions reported for the optical afterglow. The small circle is the result of registering the acquisition image of the GTC afterglow spectrum on the deep late image taken with the same telescope. The three circles are consistent with a faint underlying object which we propose to be the host galaxy. Lower panel: the GRB 120327A I-band light curve from IAC80.

calibration was carried out using 21 stars from the USNO-B1.0 catalog yielding a dispersion of 0.16 mag (not included in the error of the above two measurements). We note that the last three IAC80 images are contemporaneous to the GTC spectra (Fig. 1).

\section{Analysis}

In this section we analyze the absorption features of the host galaxy detected in our X-shooter spectra. We report on the detected lines (Sect. 4.1), line-fitting procedure (Sect. 4.2), low, excited and high absorption features (Sects. 4.3, 4.4 and 4.5, respectively), and the hydrogen column density (Sect. 4.6). Given the low $\mathrm{S} / \mathrm{N}$ of the second epoch spectrum, this is presented in Sect. 4.7 only, to assess the variability of some absorption features.

\subsection{Detected lines}

The host galaxy of GRB 120327A is extremely rich in metal lines. Metallic features are apparent from neutral (N I, O I, Mg I, P I, Ar I, Ca I), low-ionization (C II, Mg II, Al II, Al III, Si II, P II, S II, Ca II, Cr II, Fe II, Ni II, Zn II), and high-ionization (C IV, $\mathrm{N}$ V, O VI, Si IV, P V, S IV) species. In addition, strong absorption from the fine structure levels of C II, O I, Si II, Fe II and from the metastable levels of Fe II and Ni II is identified, suggesting that the intense radiation field from the GRB excites such features. Table 2 gives a summary of all the absorption lines due to the host galaxy gas. The equivalent widths of the most representative features are reported in Appendix B. The spectral features were analyzed with FITLYMAN (Fontana \& Ballester 1995). This program can simultaneously fit several absorption lines, linking the redshifts, column densities and Doppler parameters (b) if required. The probed ISM of the host galaxy is resolved into three components separated by $\sim 40 \mathrm{~km} \mathrm{~s}^{-1}$ from each other, which contribute to the absorption system. The wealth of metalline transitions allows us to precisely determine the redshift of the GRB host galaxy, $z=2.8145 \pm 0.0001$, setting the reference point to the central component. This component features the strongest absorption from the excited levels of the intervening gas with respect to the corresponding ground state levels, implying that it is the closest to the GRB (see next Section for details). This redshift is in agreement with the values previously reported for GRB 120327A (Perley \& Tanvir 2012; Krühler et al. 2012; Sánchez-Ramirez et al. 2012).

\subsection{Line-fitting procedure}

To dissect the GRB 120327A environment into components, we select a sample of absorption lines with the following properties: i) they are in the X-shooter VIS arm (which has the highest resolution); ii) they fall in a high signal-to-noise ( $\mathrm{S} / \mathrm{N})$ wavelength region; iii) they are far away from atmospheric lines and other spurious features; iv) they are not saturated; v) they are produced by low ionization transitions (high ionization levels are analyzed separately, see Sect. 4.5). According to these criteria, we selected the following lines to guide the identification of the different components constituting the circumburst matter: Ni II $\lambda \lambda 1741,1751$, Al III $\lambda \lambda 1854,1862$, Ni II $\lambda \lambda \lambda 2166,2217$, 2223 (the last multiplet representing a metastable level of the Ni II ion).

A three-component model provides a good fit for this set of lines (see Figs. 2 and 3), and this fit fixes the redshift of the three components constituting the host galaxy absorption system. In the following, we identify component I as the bluest one, and component III as the reddest one. The velocity separation between component I and II (III) is 41 (76) $\mathrm{km} \mathrm{s}^{-1}$. We stress that the number of identified components in a GRB environment, apart from reflecting the complexity of the specific host galaxy ISM, depends on the resolution of the instrument used. The nominal resolution of the X-shooter VIS arm is $R=8800$. However, during our observations, the seeing was down at $0.5^{\prime \prime}$ for most of the integration time, and this increased the actual resolution. To estimate the true resolution of our observations, we used the width of the telluric lines, finding that $R=12000$ in the VIS and $R=7000$ in the UVB and NIR arms represent more reliable values for our data. A resolution of $R=12000$ translates into an instrumental $b$ parameter of $v_{R}=15 \mathrm{~km} \mathrm{~s}^{-1}$ in velocity space. This means that we can not resolve line separations much smaller than $v_{R}$. 
Table 2. Absorption line column densities for the three components of the main system.

\begin{tabular}{|c|c|c|c|c|}
\hline Atomic/ionic species & Observed transitions & $\mathrm{I}\left(-41 \mathrm{~km} \mathrm{~s}^{-1}\right)$ & II $\left(0 \mathrm{~km} \mathrm{~s}^{-1}\right)$ & III $\left(+35 \mathrm{~km} \mathrm{~s}^{-1}\right)$ \\
\hline $\begin{array}{l}\mathrm{HI}^{2} S_{1 / 2} \\
\mathrm{C}_{\text {III }}{ }^{2} \mathrm{P}^{0}\end{array}$ & $\begin{array}{c}\operatorname{Ly} \alpha, \operatorname{Ly} \beta, \operatorname{Ly} \gamma, \operatorname{Ly} \delta \\
\lambda 1036, \lambda 1334\end{array}$ & - & $\begin{array}{c}22.01 \pm 0.09 \\
(\mathrm{~S})^{1}\end{array}$ & - \\
\hline $\mathrm{C}_{\mathrm{III}}{ }^{2} \mathrm{P}_{3 / 2}^{1 / 2}(1 \mathrm{~s})$ & $\lambda 1037, \lambda 1335$ & $14.67 \pm 0.04$ & $14.05 \pm 0.10$ & $<13.0$ \\
\hline C IV ${ }^{2} S_{1 / 2}^{3 / 2}$ & $\lambda 1548, \lambda 1550$ & & $(\mathrm{~S})$ & \\
\hline $\mathrm{N}_{\mathrm{I}}{ }^{4} \mathrm{~S}_{3 / 2}^{0}$ & $\lambda 952, \lambda \lambda \lambda 1134$ & $16.37 \pm 0.06$ & $15.06 \pm 0.03$ & $<13.7$ \\
\hline $\mathrm{NV}^{2} \mathrm{~S}_{1 / 2}^{3 / 2}$ & $\lambda 1238, \lambda 1242$ & - & $13.56 \pm 0.03$ & - \\
\hline $\mathrm{OI}{ }^{3} \mathrm{P}_{2}$ & $\lambda 1039, \lambda 1302$ & $16.16 \pm 0.08(\mathrm{~S})$ & $15.53 \pm 0.37(\mathrm{~S})$ & $15.68 \pm 0.06(\mathrm{~S})$ \\
\hline $\mathrm{OI}^{3} \mathrm{P}_{1}(1 \mathrm{~s})$ & $\lambda 1304$ & $15.92 \pm 0.22(\mathrm{~S})$ & $14.17 \pm 0.18(\mathrm{~S})$ & $<13.8$ \\
\hline $\mathrm{O}$ I ${ }^{3} \mathrm{P}_{0}(2 \mathrm{~s})$ & $\lambda 1306$ & $14.38 \pm 0.04$ & $14.30 \pm 0.05$ & $<13.8$ \\
\hline${ }^{O}$ VI ${ }^{2} \mathrm{~S}_{1 / 2}$ & $\lambda 1031, \lambda 1037$ & & (S) & \\
\hline $\operatorname{Mg}{ }^{1}{ }^{1} S_{0}$ & $\lambda 2026, \lambda 2852$ & $15.29 \pm 0.03$ & $14.01 \pm 0.13$ & $12.45 \pm 0.02$ \\
\hline $\mathrm{Mg}$ II ${ }^{2} \mathrm{~S}_{1 / 2}$ & $\lambda 1239, \lambda 1240, \lambda 2796, \lambda 2803$ & $16.28 \pm 0.02$ & $15.03 \pm 0.22$ & $(\mathrm{~S})$ \\
\hline $\mathrm{Al} \mathrm{II}^{1} \mathrm{~S}_{0}$ & $\lambda 1670$ & $15.39 \pm 0.15(\mathrm{~S})$ & $16.42 \pm 0.08(\mathrm{~S})$ & $13.26 \pm 0.05(\mathrm{~S})$ \\
\hline $\mathrm{Al} \mathrm{IIII}^{2} \mathrm{~S}_{1 / 2}$ & $\lambda 1854, \lambda 1862$ & $13.21 \pm 0.02$ & $13.12 \pm 0.05$ & $12.55 \pm 0.11$ \\
\hline $\mathrm{Si}$ II ${ }^{2} \mathrm{P}_{1 / 2}^{0}$ & $\begin{array}{c}\lambda 1020, \lambda 1190, \lambda 1193, \lambda 1260 \\
\lambda 1304, \lambda 1526, \lambda 1808\end{array}$ & $15.97 \pm 0.02$ & $15.78 \pm 0.03$ & $15.78 \pm 0.03$ \\
\hline $\mathrm{Si} \mathrm{II}{ }^{2} \mathrm{P}_{3 / 2}^{0}(1 \mathrm{~s})$ & $\lambda 1264, \lambda 1309, \lambda 1533, \lambda 1816$ & $14.43 \pm 0.01$ & $15.19 \pm 0.23$ & $<13.5$ \\
\hline Si IV ${ }^{2} S_{1 / 2}$ & $\lambda 1393, \lambda 1402$ & - & $14.16 \pm 0.04$ & - \\
\hline $\mathrm{PI}^{4} \mathrm{~S}_{3 / 2}^{0}$ & $\lambda 1674$ & $13.80 \pm 0.10$ & $<13.7$ & $<13.7$ \\
\hline $\mathrm{P}_{\mathrm{II}}{ }^{3} \mathrm{P}_{0}^{3 / 2}$ & $\lambda 1152$ & $13.61 \pm 0.04$ & $13.70 \pm 0.04$ & $<13.1$ \\
\hline $\mathrm{PV}^{2} \mathrm{~S}_{1 / 2}$ & $\lambda 1117$ & - & $13.42 \pm 0.04$ & - \\
\hline $\mathrm{S}_{\text {III }}{ }^{4} \mathrm{~S}_{3 / 2}^{0}$ & $\lambda 1250, \lambda 1253, \lambda 1259$ & $15.57 \pm 0.02$ & $15.12 \pm 0.04$ & $14.67 \pm 0.04$ \\
\hline $\mathrm{S} \mathrm{VI}{ }^{2} \mathrm{~S}_{1 / 2}^{3 / 2}$ & $\lambda 944$ & - & $14.21 \pm 0.03$ & - \\
\hline $\operatorname{Ar} I{ }^{1} \mathrm{~S}_{0}$ & $\lambda 1048, \lambda 1066$ & $14.60 \pm 0.04$ & $14.32 \pm 0.04$ & $13.59 \pm 0.07$ \\
\hline $\mathrm{CaI}{ }^{1} \mathrm{~S}_{0}$ & $\lambda 4227$ & $12.14 \pm 0.02$ & - & $<11.1$ \\
\hline Ca II ${ }^{2} \mathrm{~S}_{1 / 2}$ & $\lambda 3934, \lambda 3969$ & $15.21 \pm 0.03$ & $14.01 \pm 0.13$ & $<12.7$ \\
\hline Cr II a ${ }^{6} S_{5 / 2}$ & $\lambda 2056, \lambda 2062, \lambda 2066$ & $13.89 \pm 0.02$ & $13.75 \pm 0.04$ & $13.16 \pm 0.07$ \\
\hline Fe II a ${ }^{6} D_{9 / 2}$ & $\begin{array}{c}\lambda 1081, \lambda 1125, \lambda 1143, \lambda 1144 \\
\lambda 1608, \lambda 1611, \lambda 2249, \lambda 2260 \\
\lambda 2344, \lambda 2374, \lambda 2382\end{array}$ & $15.42 \pm 0.03$ & $15.44 \pm 0.02$ & $14.03 \pm 0.11$ \\
\hline Fe II a ${ }^{6} D_{7 / 2}(1 s)$ & $\lambda 1618, \lambda 1621, \lambda 2389, \lambda 2396$ & $13.85 \pm 0.04$ & $14.09 \pm 0.03$ & $<12.8$ \\
\hline Fe II a ${ }^{6} D_{5 / 2}(2 s)$ & $\lambda 1629, \lambda 1631, \lambda 2328, \lambda 2405$ & $13.55 \pm 0.09$ & $13.87 \pm 0.04$ & $<13.4$ \\
\hline Fe II a ${ }^{6} D_{3 / 2}(3 s)$ & $\lambda 1636, \lambda 2405, \lambda 2407, \lambda 2411$ & $13.37 \pm 0.07$ & $13.55 \pm 0.07$ & $<12.9$ \\
\hline Fe II a ${ }^{6} D_{1 / 2}(4 s)$ & $\lambda 1639, \lambda 2411, \lambda 2414$ & $12.81 \pm 0.26$ & $13.16 \pm 0.12$ & $<12.8$ \\
\hline $\mathrm{Fe}$ II a ${ }^{4} \mathrm{~F}_{9 / 2}(5 \mathrm{~s})$ & $\begin{array}{l}\lambda 1566, \lambda 1612, \lambda 1637 \\
\lambda 1658, \lambda 1696, \lambda 1702\end{array}$ & $13.81 \pm 0.02$ & $13.83 \pm 0.02$ & $<13.2$ \\
\hline Fe II a ${ }^{4} F_{7 / 2}(6 s)$ & $\begin{array}{c}\lambda 1563, \lambda 1580, \lambda 1625 \\
\lambda 1659, \lambda 1712\end{array}$ & $12.82 \pm 0.18$ & $13.41 \pm 0.06$ & $<12.4$ \\
\hline Fe II a ${ }^{4} D_{7 / 2}(9 s)$ & $\lambda 1635, \lambda 2740$ & $<12.8$ & $13.05 \pm 0.16$ & $<12.8$ \\
\hline Ni II ${ }^{2} \mathrm{D}_{5 / 2}$ & $\begin{array}{l}\lambda 1317, \lambda 1370, \lambda 1454 \\
\lambda 1709, \lambda 1741, \lambda 1751\end{array}$ & $14.22 \pm 0.03$ & $13.86 \pm 0.05$ & $13.80 \pm 0.06$ \\
\hline $\mathrm{Ni}$ II ${ }^{4} \mathrm{~F}_{9 / 2}(2 \mathrm{~s})$ & $\lambda 2166, \lambda 2217, \lambda 2223, \lambda 2316$ & $13.89 \pm 0.04$ & $13.42 \pm 0.05$ & $<12.8$ \\
\hline $\mathrm{Zn} \mathrm{II}{ }^{2} \mathrm{~S}_{1 / 2}$ & $\lambda 2026, \lambda 2062$ & $13.21 \pm 0.03$ & $12.86 \pm 0.07$ & $12.23 \pm 0.46$ \\
\hline
\end{tabular}

Notes. All values of the column densities are logarithmic (in $\mathrm{cm}^{-2}$ ). Upper limits are given at the $90 \%$ confidence level. ${ }^{(1)}$ (S) marks saturated transitions and unreliable column densities.

The redshifts of our three components were fixed in order to fit the other species present in the spectrum with the same model (high ionization lines are analyzed in a different way, see Sect. 4.3). The Doppler parameter $b$ was fixed between ions and levels of the same atom and was allowed to vary between different atoms. $b$ is in the range $15-40 \mathrm{~km} \mathrm{~s}^{-1}, 5-30 \mathrm{~km} \mathrm{~s}^{-1}$ and $25-100 \mathrm{~km} \mathrm{~s}^{-1}$ for components I, II and III respectively. In some cases the fit returned a $b$ value for component II smaller than the spectral resolution. To assess the robustness of the column density values for these specific lines, we verified that the column density obtained fixing $b$ to $15 \mathrm{~km} \mathrm{~s}^{-1}$ was within $1 \sigma$ with respect to that obtained leaving $b$ free to vary. Table 2 summarizes all the species and the corresponding transitions identified in our spectrum. The reported uncertainties are the formal $1 \sigma$ errors provided by FITLYMAN, while the upper limits are at the $90 \%$ confidence level. The next sub-sections describe the detected lines in detail.

\subsection{Low ionization lines}

We briefly describe here all transitions due to neutral, singly- or doubly-ionized species. The C II $\lambda 1334$, O I $\lambda 1039$, O I $\lambda 1302$ and Al II $\lambda 1670$ transitions are heavily saturated, thus the computed column densities of these species are unreliable or should be regarded with caution (they are marked with an " $\mathrm{S}$ " in Table 2). In addition, some level of saturation might also be present in component I of the Si II $\lambda 1808$ line, the only one available to determine the Si II column density. We note that the $\mathrm{Mg}$ II $\lambda 2796$ and $\mathrm{Mg}$ II $\lambda 2803$ lines are heavily saturated too, 


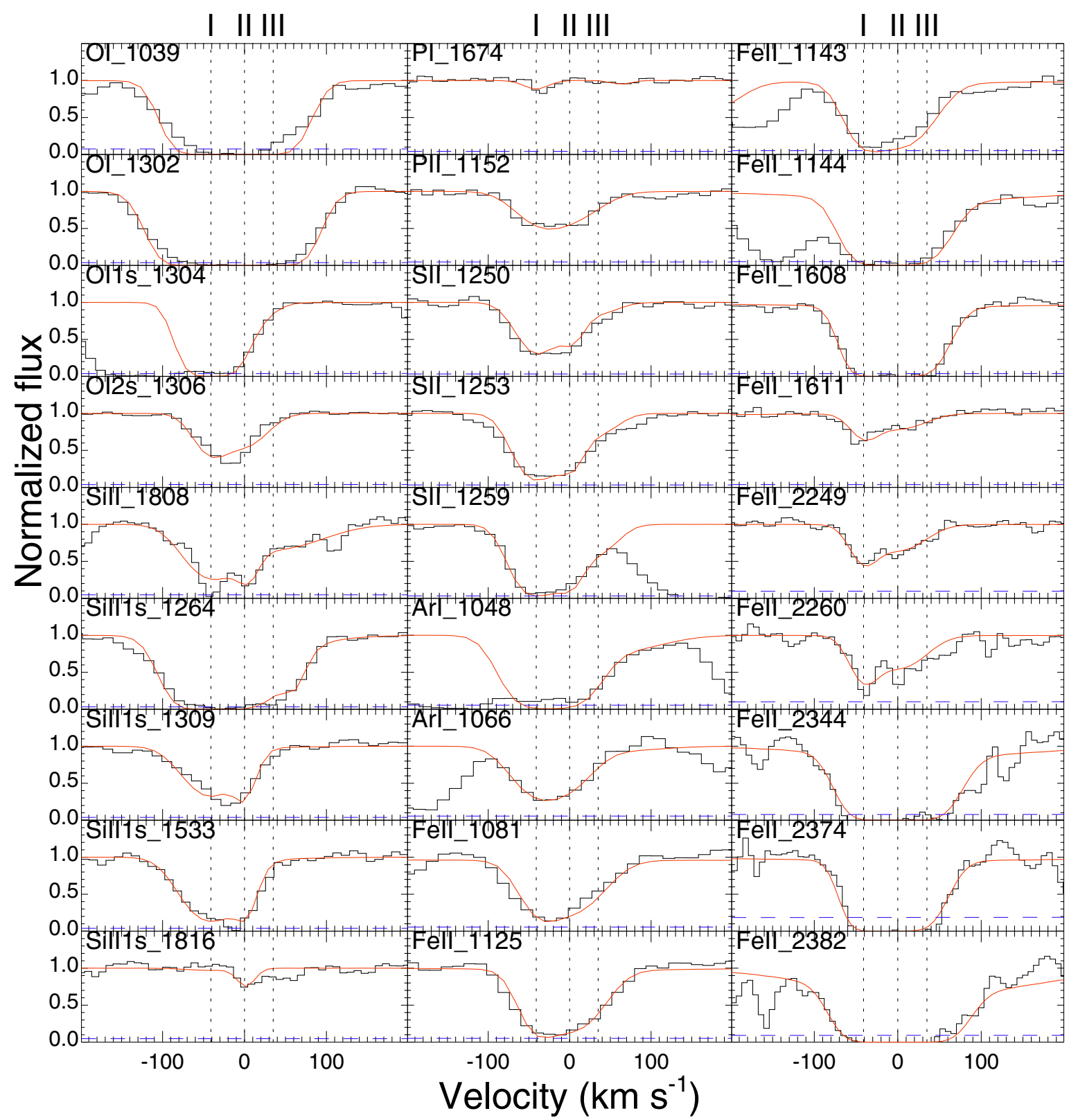

Fig. 2. GRB 120327A absorption features. Solid, red lines represent the best-fit model to the three Voigt components. Vertical dashed lines identify the component velocities. Blue, dashed lines display the error spectrum. The zero point has been arbitrarily placed at the redshift of the second component $(z=2.8145)$.

but we can determine the $\mathrm{Mg}$ II column density using the weak $\operatorname{Mg}$ II $\lambda \lambda 1239,1240$ doublet. Figures 2-4 show all the transitions used to determine the column densities, and the corresponding best-fit models. In particular, Figs. 2 and 3 display transitions observed in the UVB and VIS arms, while Fig. 4 shows NIR arm transitions, where the resolution is lower and the components are not well separated. In each figure the three components identified by our analysis are marked by vertical dashed lines.

All singly and doubly ionized features, with the excpetion of P II and Ca II, are detected in each of the three components. It is evident, however, that component III is the least populated of the three, while components I and II show similar column densities, with the exception of Ca II, which is stronger in component I.

The neutral species (N I, O I, Mg I, P I, Ar I, Ca I) are considerably weaker in component III (if detected). In addition, P I and Ca I show absorption only in component I, and the N I and Mg I column densities of component I are considerably higher than that of component II. The strong saturation of the O I features prevents similar considerations for this species.

\subsection{Excited levels}

The level structure of an atom or ion is characterized by a principal quantum number $n$, which defines the atomic level, and by the spin-orbit coupling (described by the quantum number $j$ ), which splits these levels into fine structure sublevels. In GRB absorption spectra, several excited features are detected at the GRB redshift, due to the population of both $n>1$ and/or $n=1$ fine structure levels. GRB 120327A is not an exception, featuring excited levels of C II, O I, Si II, Fe II and Ni II. In particular, $\mathrm{C}$ II and Si II show fine structure lines produced by the first excited $n=1$ level, while O I shows fine structure lines from the first and second excited $n=1$ levels (Figs. 2 and 3). Ni II features instead absorption from the ground level of the $n=2$ quantum 


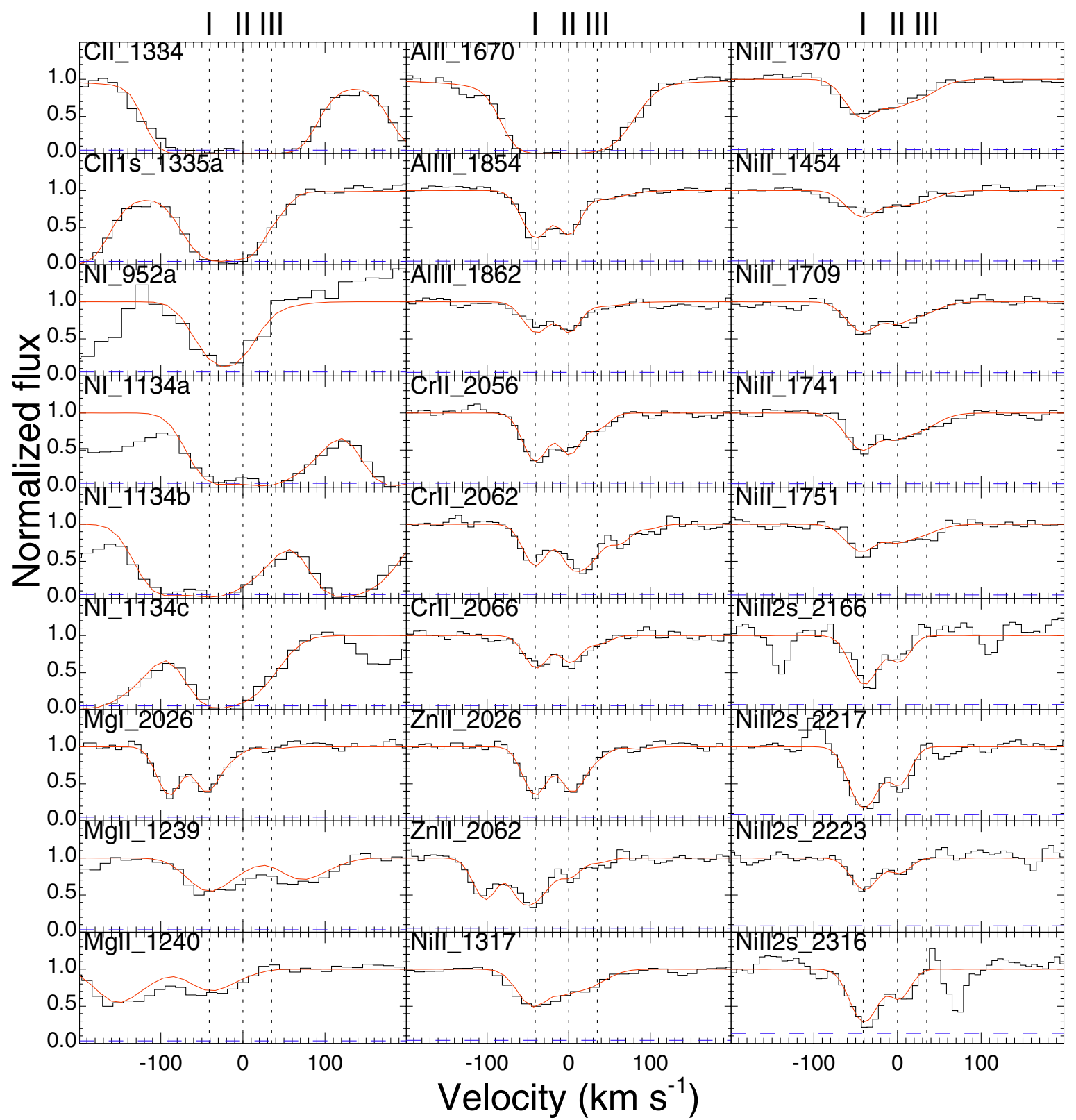

Fig. 3. GRB 120327A absorption features. Solid, red lines represent the best-fit model to the three Voigt components. Vertical dashed lines identify the component velocities. Blue, dashed lines display the error spectrum. The zero point has been arbitrarily placed at the redshift of the second component $(z=2.8145)$. The absorption on the left of the Mg I $\lambda 2026$ line is due to $\mathrm{Zn}$ II $\lambda 2026$.

state (Fig. 3). Finally, Fe II shows a plethora of absorption lines due to at least seven excited levels belonging to the $n=1, n=2$ and $n=3$ quantum states (Fig. 5).

The excited transitions displayed in Figs. 2, 3 and 5 are marked with a number that indicates the level above the $n=1$ fundamental state to adopt a compact notation (see Table 2 for the standard definition based on their quantum numbers). For example, C IIIs is one level above the fundamental state and so on. Ni II2s represents the ground state level of the $n=2$ quantum state for the singly-ionized Nickel, while Fe II5s and Fe II9s are the ground state levels of the $n=2$ and $n=3$ quantum states for the singly-ionized Iron, respectively.

As for the low ionization lines, saturation problems arise when one tries to fit the C II and O I excited lines. In particular, the OI1s $\lambda 1304$ fine structure line is heavily blended with the Si II $\lambda 1304$ feature. The computation of the corresponding column density has been attempted by simultaneously fitting the
O I1s $\lambda 1304$ and Si II $\lambda 1304$ lines, fixing the column densities of the latter to that evaluated using non-saturated transitions. Despite this, we cannot exclude strong saturation effects, so the computed column densities of the C II and O I fine structure levels should be considered with caution.

Excited levels are not present in component III, just like neutral species. However, while the latter tend to populate more heavily component I (see Table 2 and previous sub-section), the former are more prominent in component II, when compared to their corresponding ground state level (with the exception of Ni II2s and Fe II5s).

\subsection{High-ionization lines}

The high-ionization lines detected in the GRB 120327A spectrum belong to the following species: C IV, N V, O VI, Si IV, P V and S VI. We prefer to adopt a different approach while fitting 


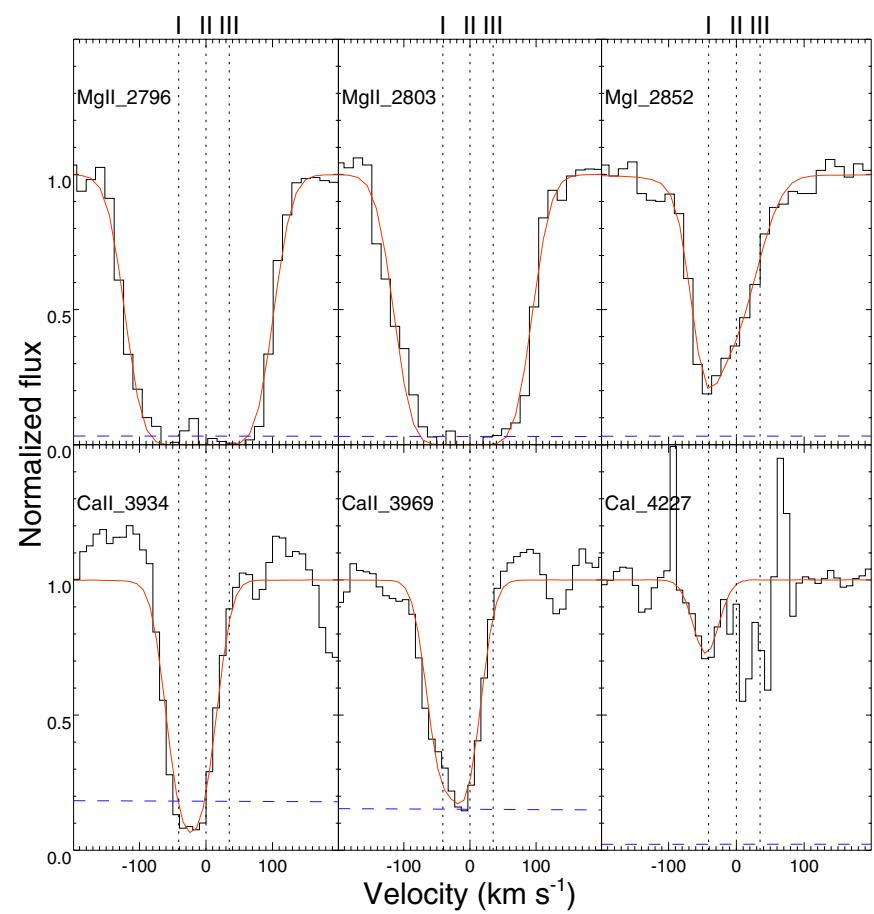

Fig. 4. GRB 120327A absorption features detected in the X-shooter NIR arm. Solid, red lines represent the best-fit model to the three Voigt components. Blue, dashed lines display the error spectrum. Vertical dashed lines identify the component velocities. Note that the resolution in this arm is lower than in the VIS one, so that single components do not appear well separated.

high ionization lines because i) some of them appear to be saturated (in particular the C IV and O VI features); ii) most of them fall in the X-shooter UVB arm, where resolution is lower; iii) a physical separation between low- and high- ionization species, and thus different line profiles, is not unexpected, because they may trace different ISM regions because of their different ionization potentials. For these reasons, we try to fit the high-ionization lines with a single Voigt profile with linked central wavelength. There is a good alignment between all the absorption lines, with the exception of the $\mathrm{N} v$ features. For this doublet, we have to shift the central wavelength to obtain a satisfactory fit.

Figure 6 shows the high-ionization lines and the best-fit model. In the last panel the Al III $\lambda 1854$ low-ionization line is shown for comparison. The vertical dotted lines mark the central velocity derived for the $\mathrm{N} v$ features (right lines) and that for all the other high-ionization species (left lines). N v appears perfectly lined-up with component II of the low-ionization species, while the central wavelength of the other high-ions falls exactly halfway between component I and II.

\subsection{Hydrogen column density}

The GRB 120327A redshift is high enough to allow several hydrogen lines belonging to the Lyman series to enter the $\mathrm{X}$-shooter spectral window. $\operatorname{Ly} \alpha$ and $\operatorname{Ly} \beta$ are clearly seen at $\sim 4650$ and $3900 \AA$, respectively. Given the ADC problem in the X-shooter observation of GRB 120327A, the normalization around the Ly $\alpha$ is not easy, since it is difficult to separate the contribution of the continuum to that of the Ly $\alpha$ wings. On the other hand, the fitting of Lyman lines bluewards of the Ly $\alpha$ must be performed with caution, since the Ly $\alpha$ forest affects relevant contamination to the line profiles. We thus used two different approaches to determine the hydrogen column density, and checked the consistency of the corresponding results. To fit the Ly $\alpha$, we first extracted the spectrum from the two dimensional one using a standard response curve for the fluxing. We then dereddened it for Galactic extinction and normalized with a power-law $F_{\lambda} \propto \lambda^{-0.3}$. A Ly $\alpha$ feature was then fitted to this normalized spectrum. We note that this spectral index is not consistent with that deduced from photometric measures (Sect. 5.2) or from the GTC spectrum (Sect. 5.5), due to the ADC problem. Figure 7 (top panel) shows the result of the fit with $\log \left(N_{\mathrm{H}, 1} / \mathrm{cm}^{-2}\right)=22.00 \pm 0.10 \mathrm{~cm}^{-2}$. Dotted lines delimit the $1 \sigma$ uncertainty. To study other Lyman features, we normalized the spectrum locally, using the few line-free regions to determine the continuum contribution, and simultaneously fit the $\operatorname{Ly} \beta$, Ly $\gamma$ and $\operatorname{Ly} \delta$, fixing the redshift at that of the central metallic component (i.e., $z=2.8145$ ). Bluer Lyman features are present (up to at least the $\operatorname{Ly} \theta$ ) but the $\mathrm{S} / \mathrm{N}$ in the corresponding regions is too low to enable a good fit. Figure 7 (bottom panel) shows the result of the fit with $\log \left(N_{\mathrm{H}, 2} / \mathrm{cm}^{-2}\right)=22.05 \pm 0.20$. The two results are consistent, and the best value for the hydrogen column density is obtained with a weighted average of them, yielding $\log \left(N_{\mathrm{H}} / \mathrm{cm}^{-2}\right)=22.01 \pm 0.09$. All the Lyman features are damped and cannot be separated into components, as we did for the metallic lines. The line fits are virtually insensitive to the adopted $b$ parameter.

\subsection{Second-epoch spectroscopy}

A second $\mathrm{X}$-shooter spectrum was acquired $\approx 30 \mathrm{hr}$ after the GRB. Unfortunately, the $\mathrm{S} / \mathrm{N}$ for this observation is extremely poor (see Table 1), since the afterglow was considerably fainter. However, we could compare the strongest Fe II ground state transitions between the two epochs. Columns 2 and 3 of Table 3 indicate whether the corresponding transition in Col. 1 is blended with Fe II fine structure lines or contaminated by atmospheric lines. Columns 4 and 5 report the rest-frame equivalent widths (EWs) of the transitions in the first and second epoch, respectively. Columns 6 and 7 give the variation and significance between the two observations, respectively. All lines not contaminated by telluric features show no variation at more than the $3 \sigma$ level. For the Fe II $\lambda 2344$, Fe II $\lambda 2374$ and Fe II $\lambda 2382$ features the reported variation is possibly induced by a strong contamination by sky lines. In fact, after applying the proper telluric correction, the discrepancy between the two epochs falls below the $3 \sigma$ level. Thus, at the $3 \sigma$ confidence level, the Fe II ground state is not varying. We applied the same procedure to the Fe II fine structure line with the strongest oscillator strength, i.e., the Fe IIIs $\lambda 2396$ feature. In this case, the EW in the second epoch is compatible with zero, and the significance of the variation with respect to the first epoch is more than $3 \sigma$ (Tab. 3). A simple inspection of the two spectra reveals that all the excited Fe II lines disappeared in the second observation. However, the $3 \sigma$ confidence level is reached for the Fe II $1 \mathrm{~s} \lambda 2396$ feature only. Using FITLYMAN, we could determine a $90 \%$ upper limit to its column density of $\log \left(N / \mathrm{cm}^{-2}\right)<13.5$.

\section{Results}

In this section we extract the physical information from the column densities estimated through our analysis. In Sect. 5.1 we derive the metallicity for the host galaxy of GRB 120327A; in Sect. 5.2 we compute the absorber-GRB distance comparing the data with photo-excitation codes; the characterization of the GRB 120327A host is presented in Sect. 5.3 through the analysis 


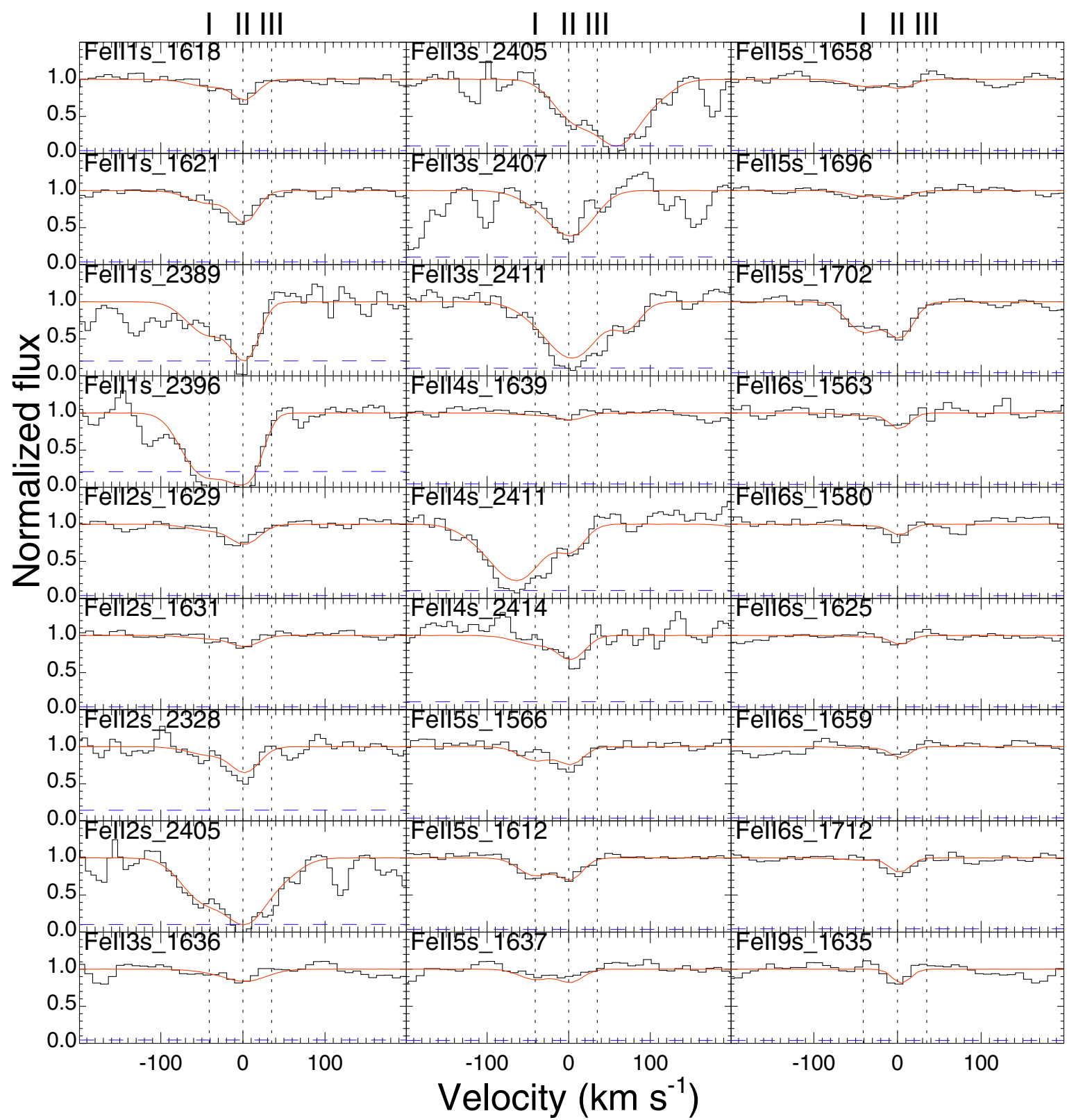

Fig. 5. Fe II excited absorption features in the GRB 120327A afterglow spectrum. Solid, red lines represent the best-fit model with three Voigt components. Blue, dashed lines display the error spectrum. Vertical dashed lines identify the component velocities. The zero point has been arbitrarily placed at the redshift of the second component $(z=2.8145)$.

of the dust depletion patterns and chemical evolution models; in Sect. 5.4 we estimate the $\mathrm{H}_{2}$ molecule column density and the corresponding molecular fraction; finally, in Sect. 5.5 we derive the extinction curve shape for the GRB 120327A afterglow.

\subsection{Abundances}

We compute here the metal abundances (relative to hydrogen) of the GRB 120327A host, both in absolute terms and compared with solar values, using the data reported in Table 2. The wide spectral coverage of $\mathrm{X}$-shooter allows this computation for several elements. We proceeded as follows. First, we summed the column densities of the transitions belonging to the same atom (different ionization and excitation states), in order to evaluate the atomic column densities for each of the three components (we consider neutral and low-ionization state only); second, since the Lyman features cannot be resolved into components, we summed up the atomic column densities of the three components to obtain the total column density for each species. We note that our (nonsaturated) column densities are always dominated by a single ionic species, with the only exception of Phosphorus, where P I and P II give a comparable contribution to the total column density.

The results are listed in Table 4 . Columns 2-4 report the total column densities for each element in components I, II and III, respectively. The total column density (obtained summing up the previous three columns) is given in Col. 5. Column 6 reports the total abundances relative to hydrogen in the GRB 120327A host, while Col. 7 reports the element abundances in the solar photosphere as published by Asplund et al. (2009). Finally, Col. 9 gives the abundances of the GRB 120327A host relative to the solar values. We derived metallicity values with respect to the solar ones between $1.9 \times 10^{-2}$ and $7.8 \times 10^{-2}$ for most 
Table 3. Comparison between the observed Fe II equivalent widths in the first and second X-shooter observation

\begin{tabular}{lcccccc}
\hline \hline Feature & Blended? & Atm? & $E W 1(\AA)$ & $E W 2(\AA)$ & Variation $(\AA)$ & Significance $(\sigma)$ \\
\hline Fe II $\lambda 1608$ & No & No & $3.11 \pm 0.04$ & $3.3 \pm 0.9$ & $+0.19 \pm 0.94$ & +0.2 \\
Fe II $\lambda 2250$ & No & No & $1.22 \pm 0.05$ & $1.54 \pm 0.17$ & $+0.32 \pm 0.22$ & +1.5 \\
Fe II $\lambda 2261$ & No & No & $1.58 \pm 0.06$ & $1.81 \pm 0.23$ & $+0.23 \pm 0.29$ & +0.8 \\
Fe II $\lambda 2344$ & Yes & Yes & $5.41 \pm 0.11$ & $3.4 \pm 0.2$ & $-2.01 \pm 0.31$ & -6.5 \\
Fe II $\lambda 2374$ & No & Yes & $4.11 \pm 0.06$ & $3.05 \pm 0.18$ & $-1.06 \pm 0.24$ & -4.4 \\
Fe II $\lambda 2382$ & Yes & Yes & $7.13 \pm 0.09$ & $4.08 \pm 0.19$ & $-3.05 \pm 0.28$ & -10.9 \\
Fe II $\lambda 2587$ & No & No & $5.81 \pm 0.29$ & $3.80 \pm 0.47$ & $-2.01 \pm 0.76$ & -2.6 \\
Fe II $\lambda 2344$ & Yes & Corrected & $4.85 \pm 0.16$ & $3.40 \pm 0.45$ & $-1.44 \pm 0.61$ & -2.4 \\
Fe II $\lambda 2374$ & No & Corrected & $3.99 \pm 0.12$ & $2.48 \pm 0.39$ & $-1.51 \pm 0.51$ & -2.9 \\
Fe II $\lambda 2382$ & Yes & Corrected & $4.71 \pm 0.16$ & $3.13 \pm 0.45$ & $-1.58 \pm 0.61$ & -2.6 \\
Fe II $\lambda 2396^{*}$ & Yes & Corrected & $1.80 \pm 0.12$ & $0.24 \pm 0.33$ & $-1.56 \pm 0.45$ & -3.5 \\
\hline
\end{tabular}

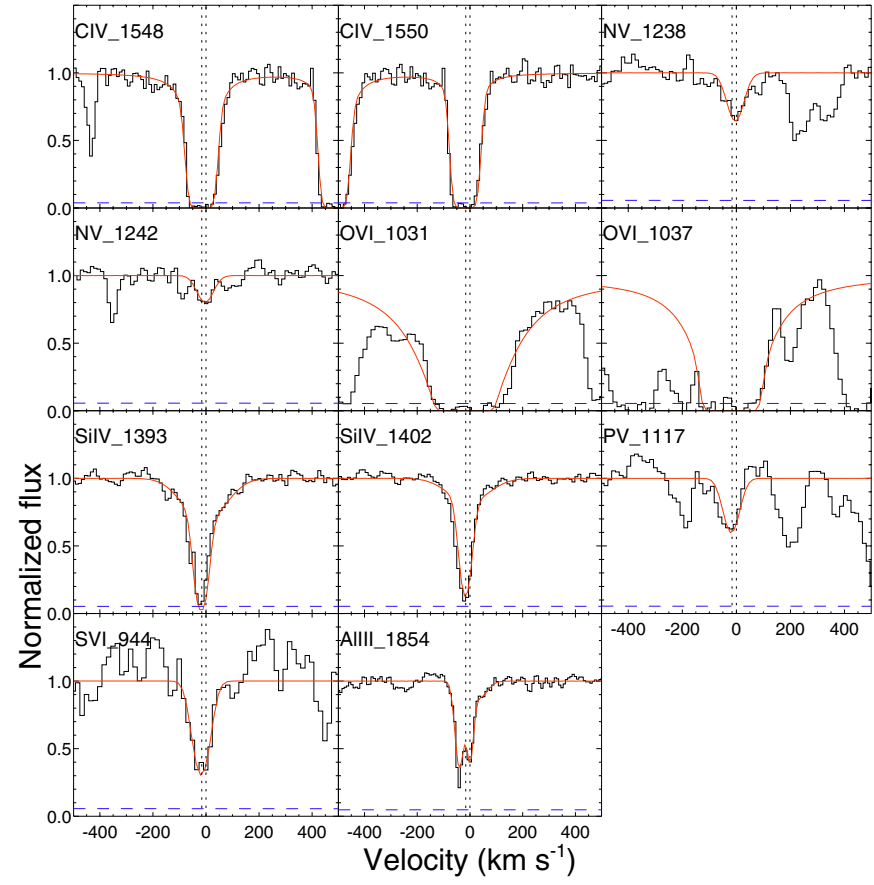

Fig. 6. GRB 120327A high ionization absorption features. Solid, red lines represent the best-fit model with a single Voigt component. Blue, dashed lines display the error spectrum. Vertical dashed lines identify the component velocities. In particular, the rightmost line indicates the center of the $\mathrm{N} \mathrm{V}$ profile, while the left one the center of all the other lines.

of the elements. Exceptions to this behavior are represented by $\mathrm{C}$ and $\mathrm{Al}$, for which Solar or supersolar values are instead obtained. This deviation is most likely due to the fact that the derived column densities of these elements suffer from strong saturation problems (as that of $\mathrm{O}$ ), so the corresponding abundances are not reliable.

\subsection{Distance between the GRB and the absorbers}

As shown in the previous section, a wealth of excited lines from C II, O I, Si II, Ni II, and especially from Fe II are detected. The strong variability in the column density of the Fe II and Ni II excited levels observed in GRB 060418 (Vreeswijk et al. 2007), GRB 080319B (D'Elia et al. 2009a), and GRB 080310 (De Cia et al. 2012) ruled out collisional processes and direct infrared pumping as responsible for the excitation. This leaves us with the indirect UV pumping as responsible for
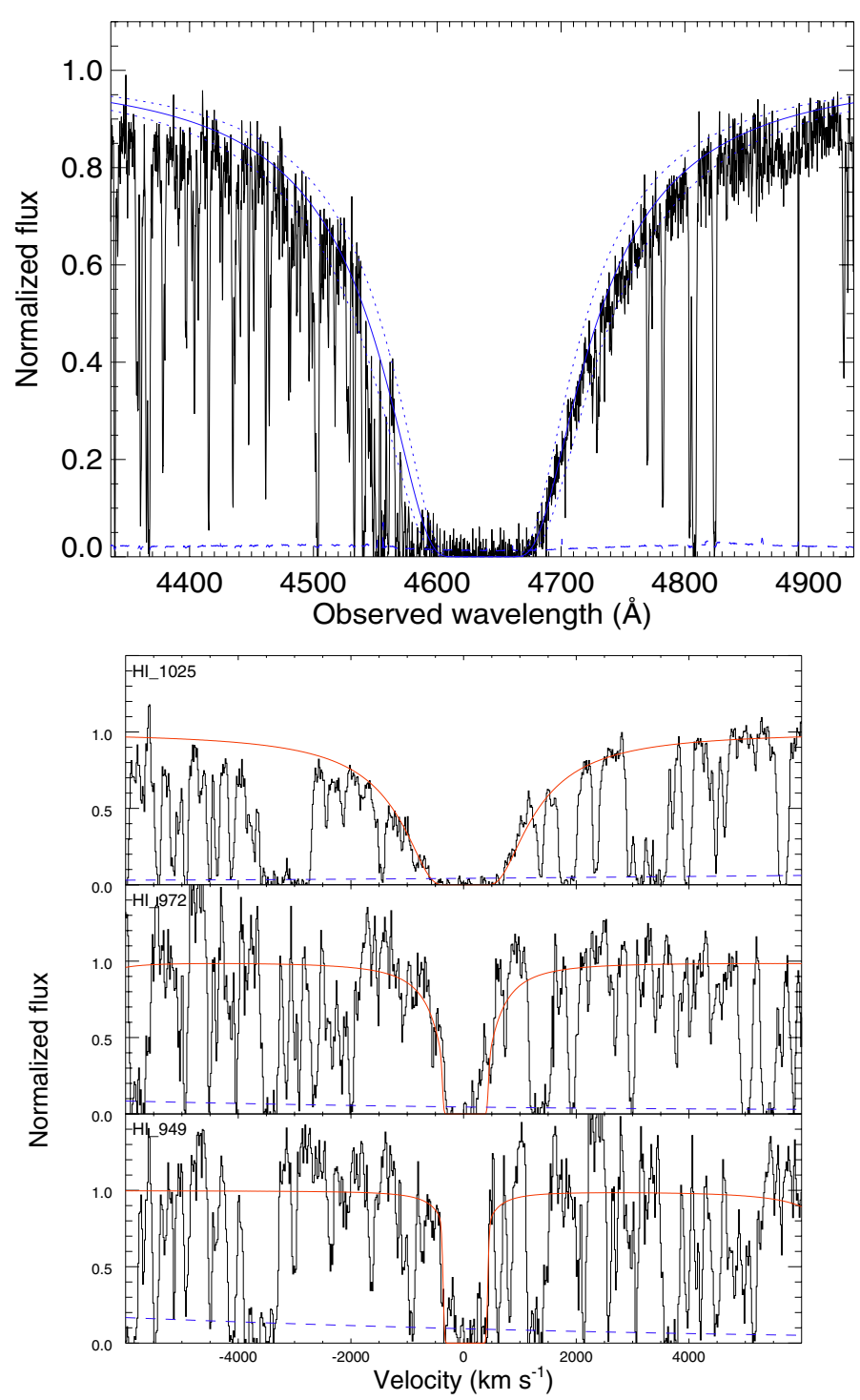

Fig. 7. Top panel: Ly $\alpha$ absorption feature at the GRB 120327A redshift. The solid, blue line represents the best-fit model to the absorption profile; the dotted, blue lines enclose the $1 \sigma$ uncertainty. Bottom panel: $\operatorname{Ly} \beta, \operatorname{Ly} \gamma$ and $\operatorname{Ly} \delta$ features (from top to bottom) and the corresponding fit result (solid, red lines). Dashed blue lines displays the error spectrum.

the excited level population (Prochaska et al. 2006). A collisional origin of the excitation can be ruled out even if multiepoch spectroscopic data are missing. In fact, collisions populate 
Table 4. Metallicities.

\begin{tabular}{|c|c|c|c|c|c|c|c|}
\hline \multirow[b]{2}{*}{ Element $X$} & \multicolumn{4}{|c|}{$\log N_{X} / \mathrm{cm}^{-2}$} & \multirow[t]{2}{*}{$\log N_{X} / N_{\mathrm{H}}$} & \multirow[t]{2}{*}{$\left(\log N_{X} / N_{\mathrm{H}}\right)_{\odot}^{a}$} & \multirow[t]{2}{*}[X/\mathrm{H}]{} \\
\hline & Component I & Component II & Component III & Total & & & \\
\hline $\mathrm{C}^{S}$ & $16.22 \pm 0.26$ & $18.65 \pm 0.15$ & $14.99 \pm 0.20$ & $18.65 \pm 0.15$ & $-3.36 \pm 0.17$ & $-3.57 \pm 0.05$ & $0.21 \pm 0.18$ \\
\hline $\mathrm{N}$ & $16.37 \pm 0.06$ & $15.06 \pm 0.03$ & - & $16.39 \pm 0.06$ & $-5.62 \pm 0.10$ & $-4.17 \pm 0.05$ & $-1.45 \pm 0.11$ \\
\hline $\mathrm{O}^{S}$ & $16.69 \pm 0.08$ & $15.57 \pm 0.33$ & - & $16.72 \pm 0.08$ & $-5.29 \pm 0.12$ & $-3.31 \pm 0.05$ & $-1.98 \pm 0.13$ \\
\hline $\mathrm{Mg}$ & $16.32 \pm 0.02$ & $15.07 \pm 0.20$ & $12.45 \pm 0.02$ & $16.34 \pm 0.02$ & $-5.67 \pm 0.09$ & $-4.40 \pm 0.04$ & $-1.27 \pm 0.10$ \\
\hline $\mathrm{Al}^{S}$ & $15.39 \pm 0.15$ & $16.42 \pm 0.08$ & $13.34 \pm 0.05$ & $16.46 \pm 0.07$ & $-5.55 \pm 0.11$ & $-5.55 \pm 0.03$ & $0.00 \pm 0.11$ \\
\hline $\mathrm{Si}$ & $15.98 \pm 0.02$ & $15.88 \pm 0.05$ & $15.78 \pm 0.03$ & $16.36 \pm 0.03$ & $-5.65 \pm 0.09$ & $-4.49 \pm 0.03$ & $-1.16 \pm 0.09$ \\
\hline $\mathrm{P}$ & $14.02 \pm 0.06$ & $13.70 \pm 0.04$ & - & $14.19 \pm 0.04$ & $-7.82 \pm 0.10$ & $-6.59 \pm 0.03$ & $-1.23 \pm 0.10$ \\
\hline S & $15.57 \pm 0.02$ & $15.12 \pm 0.04$ & $14.67 \pm 0.04$ & $15.74 \pm 0.02$ & $-6.27 \pm 0.09$ & $-4.88 \pm 0.03$ & $-1.39 \pm 0.09$ \\
\hline $\mathrm{Ar}$ & $14.60 \pm 0.04$ & $14.32 \pm 0.04$ & $13.59 \pm 0.07$ & $14.81 \pm 0.03$ & $-7.20 \pm 0.09$ & $-5.60 \pm 0.13$ & $-1.60 \pm 0.15$ \\
\hline $\mathrm{Ca}$ & $15.21 \pm 0.03$ & $14.01 \pm 0.13$ & - & $15.24 \pm 0.03$ & $-6.77 \pm 0.09$ & $-5.66 \pm 0.04$ & $-1.11 \pm 0.10$ \\
\hline $\mathrm{Cr}$ & $13.89 \pm 0.02$ & $13.75 \pm 0.04$ & $13.16 \pm 0.07$ & $14.17 \pm 0.02$ & $-7.84 \pm 0.09$ & $-6.36 \pm 0.04$ & $-1.48 \pm 0.09$ \\
\hline $\mathrm{Fe}$ & $15.45 \pm 0.03$ & $15.49 \pm 0.02$ & $14.03 \pm 0.11$ & $15.78 \pm 0.02$ & $-6.23 \pm 0.09$ & $-4.50 \pm 0.04$ & $-1.73 \pm 0.10$ \\
\hline $\mathrm{Ni}$ & $14.39 \pm 0.02$ & $13.99 \pm 0.04$ & $13.80 \pm 0.06$ & $14.61 \pm 0.02$ & $-7.41 \pm 0.09$ & $-5.78 \pm 0.04$ & $-1.63 \pm 0.09$ \\
\hline $\mathrm{Zn}$ & $13.21 \pm 0.03$ & $12.86 \pm 0.07$ & $12.23 \pm 0.46$ & $13.40 \pm 0.04$ & $-8.61 \pm 0.10$ & $-7.44 \pm 0.05$ & $-1.17 \pm 0.11$ \\
\hline
\end{tabular}

Notes. ${ }^{(a)}$ Solar abundances taken from photospheric values in Asplund et al. (2009). ${ }^{(S)}$ Abundances derived from saturated transitions, thus not reliable.

higher energy levels less than lower energy ones. For instance, the spectra of GRB 080330 (D'Elia et al. 2009b), GRB 050730 (Ledoux et al. 2009) and GRB 090926A (D'Elia et al. 2010) exhibit an $\mathrm{Fe}$ II $\mathrm{a}^{4} \mathrm{~F}_{9 / 2}$ excited state column density that is higher than several fine structure levels of the Fe II a ${ }^{6} \mathrm{D}$ ground state. This means that radiative processes are also at work for these GRBs.

For GRB 120327A we have multi-epoch spectroscopy, with the second observation following the first one by about one day. Despite the low $\mathrm{S} / \mathrm{N}$ of the second epoch spectrum, in Sect. 4.7 we reported that the Fe II $\lambda 2396$ fine structure line disappeared in this time interval with a high confidence level (Table 3). This proves that UV pumping, rather than collisions, excited the atomic species in the GRB 120327A host galaxy gas. Anyway, even if the second epoch spectroscopy were not available, the higher column density of the Fe II a ${ }^{4} \mathrm{~F}_{9 / 2}$ excited level with respect to lower-excited Fe II states would be sufficient to assess that radiative processes are at work.

It is straightforward to note that the excited levels are directly influenced by the flux impacting the intervening gas. This means that the distance between the GRB and the absorbers can be estimated from the column densities of the excited states and their ratio. To estimate this distance, we compare the observed column densities to those predicted by a photo-excitation code for the time when the spectroscopic observations were acquired. The photo-excitation code is that used by Vreeswijk et al. (2007) and D'Elia et al. (2009) ${ }^{2}$, to which we refer the reader for more details. Basically, it solves the detailed balance equation in a time-dependent way for a set of transitions involving the levels of a given species (e.g. Fe II and Si II). The equation depends on the flux level experienced by the absorbing gas. This flux is, of course, a function of the distance $d$ of the gas from the GRB explosion site, which is a free parameter of the computation and the quantity we want to calculate. The other free parameters are the initial column densities of the levels involved and the Doppler parameter of the gas itself. Since our X-shooter spectrum suffers from ADC problems, we are not able to estimate a reliable afterglow spectral index from our dataset. Thus, we used the photometry reported by Sudilovsky et al. (2013),

\footnotetext{
2 Taking the corrigendum reported in Vreeswijk et al. (2011) into account.
}

who observed the afterglow simultaneously in the $g^{\prime} r^{\prime} i^{\prime} z^{\prime} J H K$ bands with GROND (Greiner et al. 2008). Their observations were performed $\sim 40 \mathrm{~min}$ after the GRB, and the deduced spectral index is $\beta \sim 0.5$ (modeling the spectrum as $F_{v} \propto v^{-\beta}$ ), which we assume not to vary up to the time of our observations. The flux behavior before the X-shooter observations was estimated using our photometric data from the IAC80 telescope and other data from the literature (Klotz et al. 2012a, b, c; Smith \& Virgili 2012; LaCluyze et al. 2012; Malesani et al. 2012). In detail, if the flux density in the $R$ band is $F_{R}=F_{R}\left(t_{*}\right) \times\left(t / t_{*}\right)^{-\alpha}$, we have $F_{R}\left(t_{*}\right)=2.1 \times 10^{-26} \mathrm{erg} \mathrm{cm}^{-2} \mathrm{~s}^{-1} \mathrm{~Hz}^{-1}$ at $t_{*}=360 \mathrm{~s}$, with a decay index $\alpha=1.03$.

We chose to model the Fe II first, because it has a large number of excited and ground features and thus the best determination of the column densities. On the other hand, O I and C II are useless, since almost all their transitions are saturated, while $\mathrm{Ni}$ II and Si II feature just the ground state and one excited level. We assume that before the GRB takes place the Fe II ion is in its ground state, i.e., the column densities of all the excited levels are zero. At the beginning, we try to use as the initial column density of the Fe II ground state the sum of all the column densities of the Fe II levels measured in our spectrum (ground + excited). The exact values are $\log \left(N_{\mathrm{Fe} \mathrm{II}} / \mathrm{cm}^{-2}\right)=15.45 \pm 0.03$ and $\log \left(N_{\mathrm{Fe} \mathrm{II}} / \mathrm{cm}^{-2}\right)=15.49 \pm 0.02$ for components I and II, respectively. However, these values turn out to be incompatible with the ratio between the observed excited states. In particular, the predicted Fe II $a^{4} F_{9 / 2}$ column density is too high with respect to the other excited levels, for any reasonable choice of the GRB/absorber distance and Doppler parameter. For this reason, we decide to leave the initial column density as a free parameter and determine through our model the ground state column density corresponding to the excited level population. Such an approach can not be applied to the Ni II and Si II, since they feature just two levels, and the model has at least two free parameters, i.e., the initial column density and the GRB/absorber distance.

Figure 8 shows the best fit model describing the column densities estimated for component I. The best fit parameters deduced from the model at the $2 \sigma$ confidence level are the following: i) initial column density (all Fe II supposed to be in the ground state level before the GRB) $\log \left(N_{\mathrm{I}, \mathrm{Fe}} / \mathrm{cm}^{-2}\right)=$ $14.78 \pm 0.07$; ii) Doppler parameter $b_{\mathrm{I}}=30_{-10}^{+25} \mathrm{~km} \mathrm{~s}^{-1}$; 


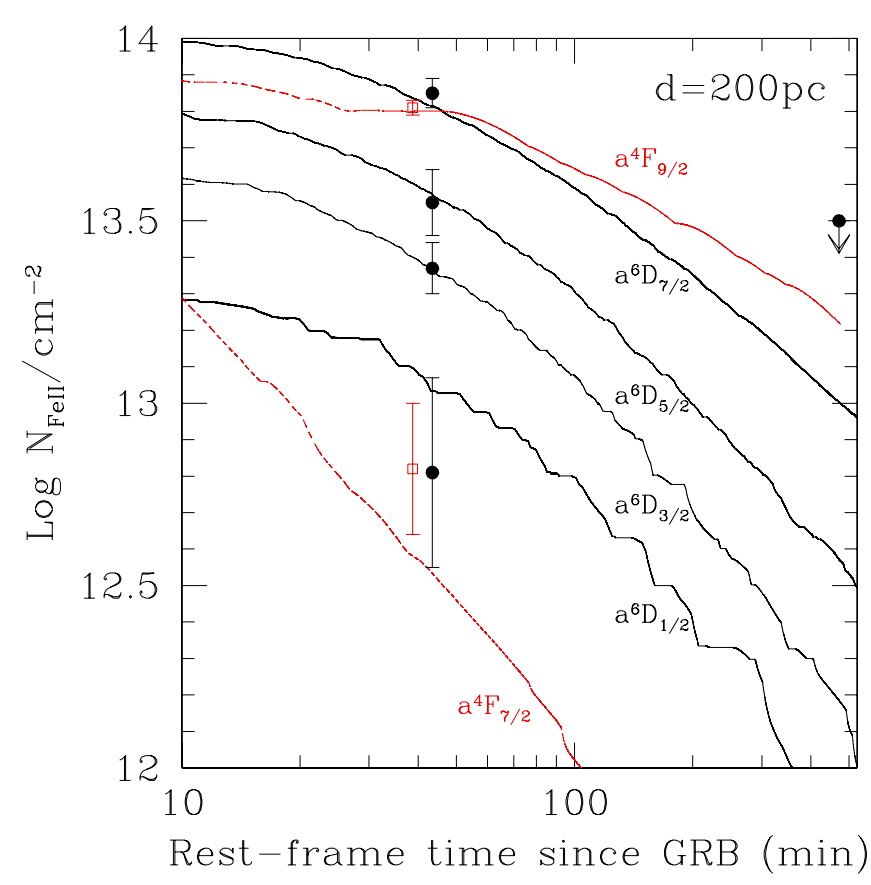

Fig. 8. Fe II column densities for the fine structure levels of the ground state (black filled circles) and of the first excited level (red open squares) for component I in the spectrum of GRB 120327A. Column density predictions from our time-dependent photo-excitation code are also shown. They refer to the fine structure levels of the ground state (solid black lines) and to the fine structure levels of the first excited level (red dashed lines), in the case of an absorber placed at $200 \mathrm{pc}$ from the GRB. The arrow represents the $90 \%$ upper limit to the column density of the first fine structure level of the ground state, computed using the second-epoch spectrum. For clarity reasons, black points and curves have been slightly offset to the right.

iii) GRB/absorber distance $d_{\mathrm{I}}=200_{-60}^{+100}$ pc. Fig. 9 describes in more details the data/model agreement showing the contour plots for component I.

A similar analysis has been performed for component II. We first try to model the whole dataset, but we find that the Fe II $\mathrm{a}^{4} \mathrm{~F}_{7 / 2}$ level drives the fit towards a high value of the reduced $\chi^{2}$, and a GRB/absorber distance which requires high column densities for the fine structure levels at later times, not in agreement with the upper limit computed from the second-epoch spectrum. Figure 10 shows the best fit model describing the column densities estimated for component II, not including the Fe II $a^{4} \mathrm{~F}_{7 / 2}$ level. The best fit parameters deduced from the model at the $2 \sigma$ confidence level are the following: i) initial column density (all Fe II supposed to be in the ground state level before the GRB) $\log \left(N_{\text {II,Fe II }} / \mathrm{cm}^{-2}\right)=14.85_{-0.07}^{+0.02}$; ii) Doppler parameter $b_{\mathrm{II}}=30_{-5}^{+15} \mathrm{~km} \mathrm{~s}^{-1}$; iii) GRB/absorber distance $d_{\mathrm{II}}=100_{-30}^{+40} \mathrm{pc}$. Figure 11 describes in more details the data/model agreement showing the contour plots for component II. If we include the $\mathrm{Fe}$ II a ${ }^{4} \mathrm{~F}_{7 / 2}$ level in the fitting procedure, the GRB/absorber distance is $d_{\mathrm{II}}=70_{-10}^{+20} \mathrm{pc}$.

\subsection{Dust depletion patterns}

The observed relative abundances of $\mathrm{Fe}$ and $\mathrm{Zn}$ are different $([\mathrm{Fe} / \mathrm{H}]=-1.73 \pm 0.10$ and $[\mathrm{Zn} / \mathrm{H}]=-1.17 \pm 0.11)$. This is usually attributed to the different refractory properties of the two elements, with the former that preferentially being locked in dust grains, and the latter free in the gas phase. The comparison
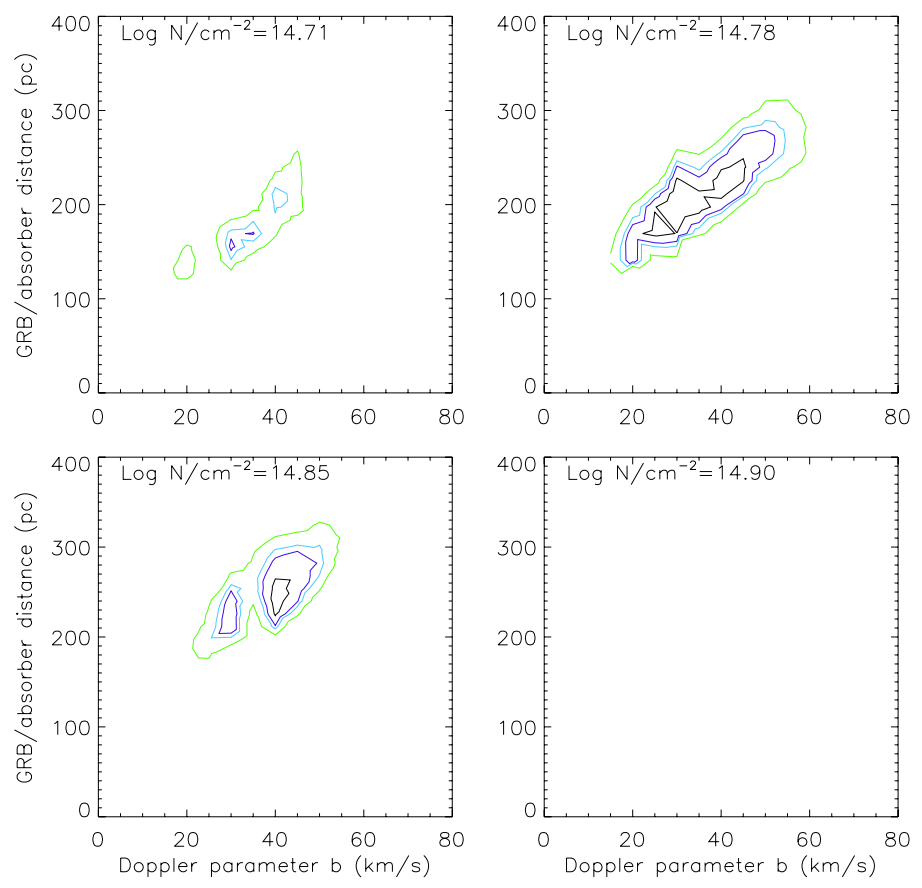

Fig. 9. Contour plots describing the data/model agreement in the computation of the distance between the GRB and component I. The four plots display the contours in the plane of Doppler parameter and GRB/absorber distance for different initial Fe II column densities. Black, violet, cyan and green contours enclose the 68\%,90\%,95\% and 99\% confidence regions, respectively. The bottom right panel is empty as at the corresponding density no combination of Doppler parameter and distance falls inside the $99 \%$ confidence surface.

between these elements is indeed a widely applied tool to derive information about the dust content along GRB and QSO lines of sight.

Dust depletion patterns can be compared to those observed in Galactic environments following a technique described by Savaglio et al. (2003). We consider the four depletion patterns observed in the Milky Way, namely, those in the warm halo (WH), warm disk + halo (WHD), warm disk (WD) and cool disk (CD) clouds (Savage \& Sembach 1996).

We find that none of the four patterns is able to reproduce or data (Fig. 12). The best fit is given by the WH cloud pattern, with a metallicity of $\log Z_{\mathrm{GRB}} / Z_{\odot} \sim-1.3$ and a GRB dust-to-metal ratio slightly lower than that of the WH environment in the Milky Way, e.g., $d / d_{\mathrm{WH}} \sim 0.7$. This metallicity value is consistent with our $[\mathrm{Zn} / \mathrm{H}]$ measurement as expected, since WH depletion pattern assumes no depletion of zinc onto dust.

We note that $\mathrm{Si}$ and $\mathrm{Mg}$ are over-abundant compared to any depletion pattern known in the Galaxy. This can be attributed to $\alpha$-element over-abundancy with important implications about the host galaxy nature as discussed in Sect. 5.3.2. (we note however that another $\alpha$-element, $\mathrm{S}$, does not appear to be overabundant).

Knowing the hydrogen column density, the metal content and the dust-to-metal ratio, it is possible to derive an independent estimate of the extinction (see e.g., Savaglio et al. 2003). Assuming an SMC (Pei 1992) extinction curve recipe we can compute $A_{V} \sim 0.22$ mag along the line of sight of GRB 120327A. This is considerably higher than the value derived in Sect. 5.5 by modeling the flux-calibrated spectra $\left(A_{V}<\right.$ $0.03 \mathrm{mag}$ ). On the other hand, hydrogen column densities close to $10^{22} \mathrm{~cm}^{-2}$ typically involve sizable extinctions (e.g. Covino et al. 2013), although the derivation of the extinction through 


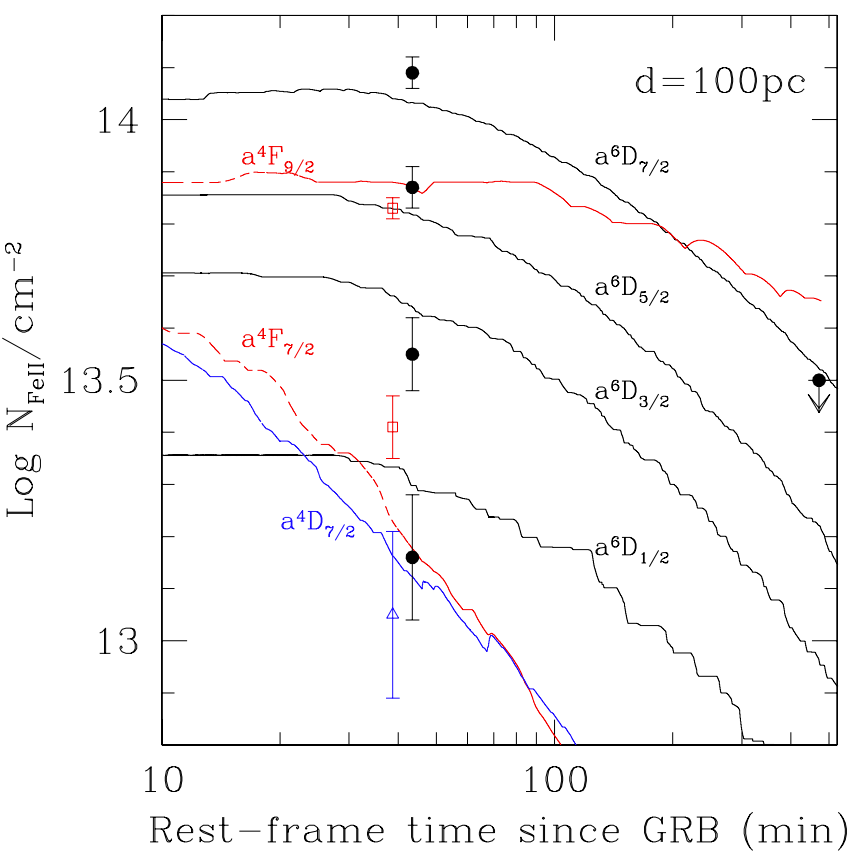

Fig. 10. Same as in Fig. 8, but for component II and an absorber placed at $100 \mathrm{pc}$. In addition, the blue, open triangle plots the second excited level of the Fe II, while the blue solid line indicates the corresponding expectation from the time-dependent photo-excitation code. For clarity reasons, black points and curves have been slightly offset to the right.

dust depletion patterns for GRB 120327A might not be reliable, given that no Galactic pattern is able to satisfactorily reproduce our data.

\subsection{Molecular absorption features}

Weak absorption lines from the Lyman and Werner bands of molecular hydrogen $\left(\mathrm{H}_{2}\right)$ are detected at $z=2.8139$ in the $\mathrm{X}$-shooter spectrum of the afterglow of GRB 120327A. The observed velocity width of the lines is similar to the instrumental resolution. We therefore constrain the $\mathrm{H}_{2}$ column densities by assuming a range of Doppler broadening parameters, between 1 and $10 \mathrm{~km} \mathrm{~s}^{-1}$, which should provide a realistic range on the total $\mathrm{H}_{2}$ column density, i.e., $\log \left(N_{\mathrm{H}_{2}} / \mathrm{cm}^{-2}\right)=15.3$ to 17.7. This corresponds to an overall molecular fraction $f=$ $2 \log N_{\mathrm{H}_{2}} /\left(\log N_{\mathrm{HI}}+2 \log N_{\mathrm{H}-2}\right)=4 \times 10^{-7}$ to $10^{-4}$.

Details on the column densities in each $\mathrm{H}_{2}$ rotational level are given in Table 5. $J=1,2$ and 3 lines are clearly detected while for $J=0$ only a few unblended lines are available and they are weak leading to more uncertain column density estimates. Figure 13 displays the two models with $b=1$ and $10 \mathrm{~km} \mathrm{~s}^{-1}$ in blue and red, respectively. In general, the present measurements are mostly indicative and should be considered with caution due to the limited resolution and $\mathrm{S} / \mathrm{N}$, and possible blending with Ly $\alpha$ forest absorption. Only high spectral resolution observations are suitable to properly resolve the $\mathrm{H}_{2}$ lines, measure accurately the column densities, and study the physical conditions in the gas (Srianand et al. 2005; Noterdaeme et al. 2007). Nevertheless, the presence of $\mathrm{H}_{2}$ in this system is firmly established. This constitutes the third clear detection of $\mathrm{H}_{2}$ absorption in a GRB afterglow spectrum (Prochaska et al. 2009, Krühler et al. 2013). It is interesting to note that the molecular lines are lined up with the bluemost component I.
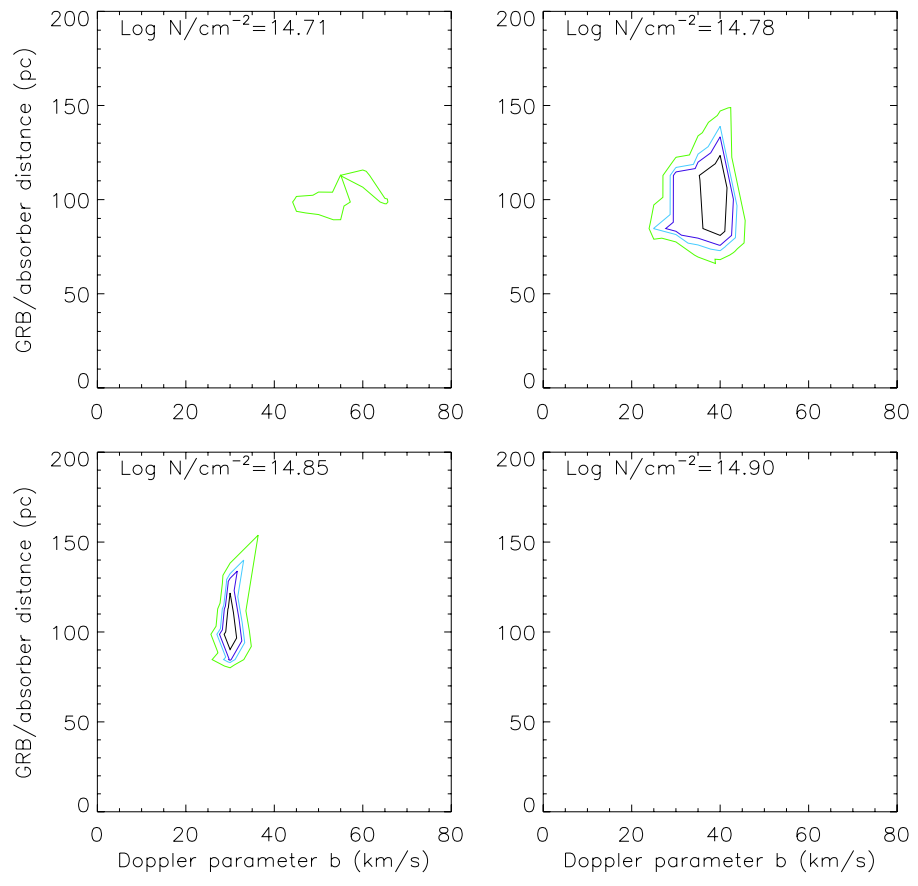

Fig. 11. Contour plots describing the data/model agreement in the computation of the distance between the GRB and component II. The four plots display the contours in the plane of Doppler parameter and GRB/absorber distance for different initial Fe II column densities. Black, violet, cyan and green contours enclose the $68 \%, 90 \%, 95 \%$ and 99\% confidence regions, respectively. The bottom right panel is empty as at the corresponding density no combination of Doppler parameter and distance falls inside the $99 \%$ confidence surface.

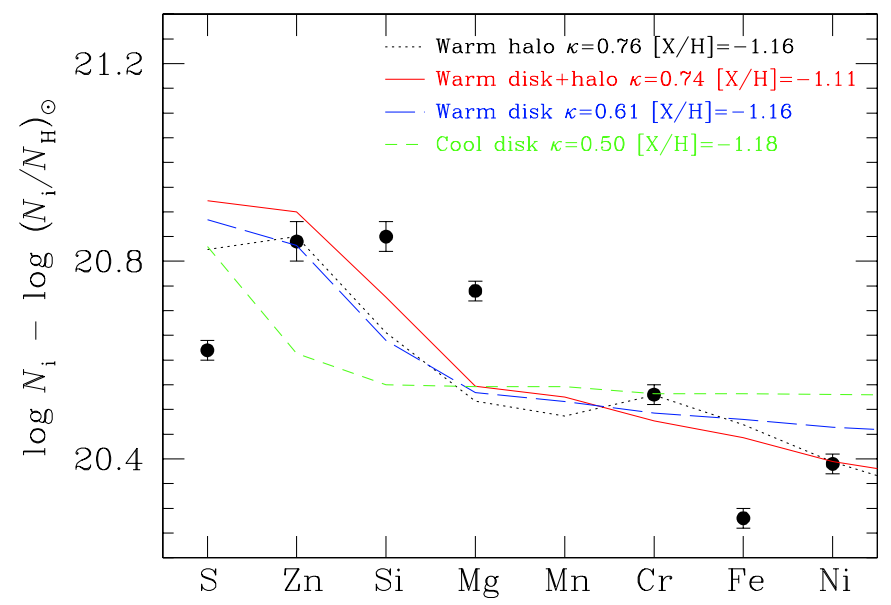

Fig. 12. Depletion patterns in the absorbing gas of GRB 120327A, taken from average gas-phase abundance measurements in warm halo (black dotted line), warm disk + halo (red solid line), warm disk (blue longdashed line) and cool disk (green short-dashed line) clouds of the Milky Way (Savage \& Sembach 1996). Filled black circles represent our data points, which are best fitted by the warm halo cloud pattern. Si and $\mathrm{Mg}$ are clearly over-abundant.

\subsection{The extinction curve shape}

The shape of extinction curves at high redshift is a powerful test for deriving information about dust formation and possibly about the various processes affecting dust absorption and destruction close to GRB sites. In most cases, inferences about dust extinction curves are obtained by modeling the spectral energy 
Table 5. $\mathrm{H}_{2}$ column densities towards GRB 120327A.

\begin{tabular}{lcc}
\hline \hline Rot. level & \multicolumn{2}{c}{$\log N_{\mathrm{H}_{2}} / \mathrm{cm}^{-2}$} \\
component & $b=1 \mathrm{~km} \mathrm{~s}^{-1}$ & $b=10 \mathrm{~km} \mathrm{~s}^{-1}$ \\
\hline$J=1$ & 17.6 & 15.1 \\
$J=2$ & 16.0 & 14.2 \\
$J=3$ & 17.1 & 14.8 \\
\hline Total & 17.7 & 15.3 \\
\hline
\end{tabular}
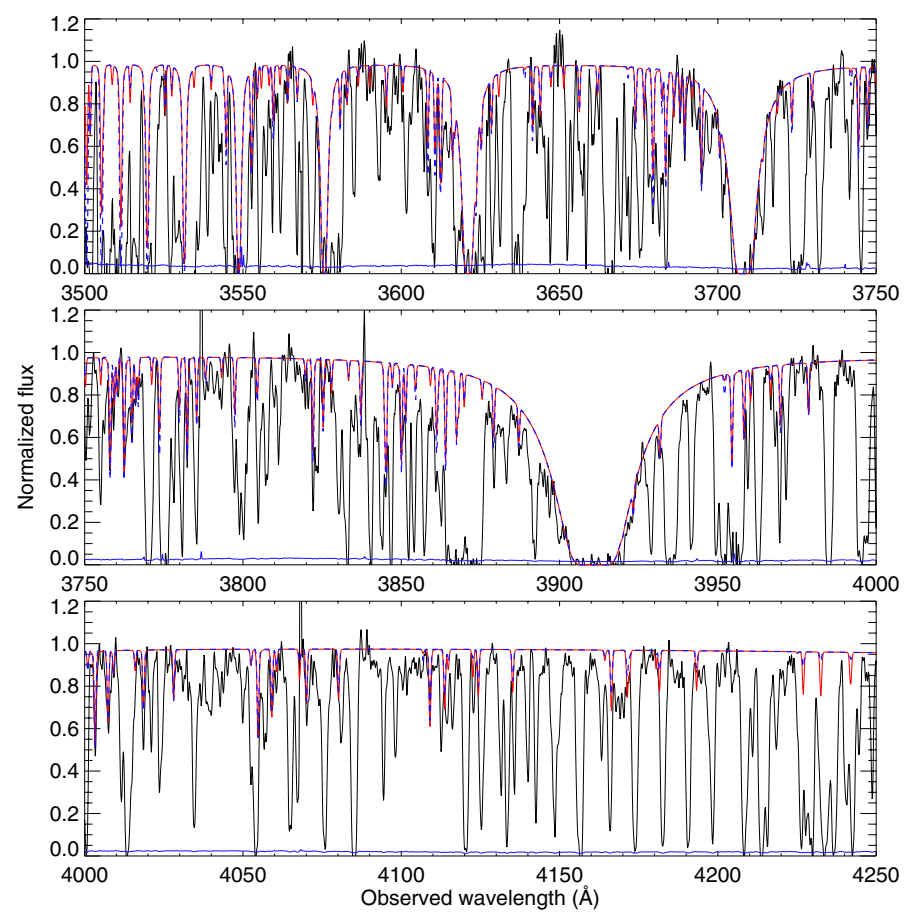

Fig. 13. Single component fit for the $\mathrm{H}_{2}$ absorption lines. The two models with $b=1$ and $b=10 \mathrm{~km} \mathrm{~s}^{-1}$ are shown in dashed blue and solid red, respectively. These models are barely distinguishable by eye, a deep zoom is necessary to catch the different profiles. The X-shooter spectrum is overimposed in black. The bottom, blue solid line displays the error spectrum.

distribution obtained through photometric observations of GRB afterglows, which unfortunately suffers from a strong degeneracy between afterglow spectral slope and extinction. Moreover, for very high redshift events in particular, uncertainties in photometry of single bands, which can also present specific calibration problems may affect the whole analysis (see for instance the discussion in Stratta et al. 2007 and Zafar et al. 2010 about GRB 050904). For early time afterglows, the lack of simultaneity (within few hours) of the available photometric information has to be properly considered to avoid spurious results.

For most GRB afterglows, when accurate multiband photometry is available (e.g., Covino et al. 2008, 2013; Schady et al. 2010; Kann et al. 2010), the derived extinction curve is in fair agreement with what is observed locally in the Small Magellanic Cloud (SMC, Pei 1992), although often, because of the limited wavelength resolution, this simply means that the observed extinction curve has to be chromatic (i.e. wavelength dependent) and featureless. A few remarkable exceptions have been recorded in an intervening system along the line of sight of GRB 060418 (Vreeswijk et al. 2007), for GRB 070802 (Krühler et al. 2008; Elíasdóttir et al. 2009) and GRB 080607 (Prochaska et al. 2009), where the characteristic absorption feature at about $2175 \AA$, prominent in the Milky Way extinction curve, has also been detected (see also Zafar et al. 2012 for a review on this feature in GRB hosts).

Clearly, when spectroscopic information is available (e.g., Watson et al. 2006; Elíasdóttir et al. 2009; Liang \& Li 2010, Zafar et al. 2011), the extinction effect and intrinsic spectral slope can be better disentangled. The case of GRB 090926A is a good example of the X-shooter capabilities in this context (D'Elia et al. 2010). Moreover, a paper exploiting the full X-shooter GRB sample to address these issues is currently in preparation (Watson et al. in prep.). In the case of GRB 120327A, however, because of the malfunctioning in the $\mathrm{X}$-shooter ADC, our flux calibration is reliable only in the NIR arm, where atmospheric dispersion is much less important and no such device is necessary. To extend our spectroscopic coverage to optical wavelengths, we used the nearly simultaneous, flux-calibrated GTC spectrum.

We removed wavelength intervals affected by telluric lines of strong absorptions. Since the Ly $\alpha$ range is important for extinction determination, we have included it in the analysis using the hydrogen column density reported in Table 2 . We rebinned the spectrum in bins of approximately $50 \AA$ by a sigma-clipping algorithm to avoid the effect of residual absorption systems. Assuming a power-law spectrum, we obtained $\beta \sim 0.5$ (consistent with the value deduced by the GROND data reported in Sudilovsky et al. 2013), where the afterglow spectrum is modeled, as is customary in GRB literature, as $F_{v} \propto v^{-\beta}$. We tried to fit the data using different extinction curves, namely, SMC, LMC and Milky Way. The best fit is obtained with an extinction of $E_{B-V}<0.01 \mathrm{mag}\left(A_{V}<0.03 \mathrm{mag}\right)$ at $95 \%$ confidence level, regardless of the adopted curve (although a higher extinction could be inferred by the metal column densities, as discussed in Watson et al.2013). We caution that possible systematics in the absolute calibrations of the various spectra involved in the analysis could affect the results well beyond the statistical uncertainties here reported.

\section{Discussion}

\subsection{The GRB 120327A line of sight}

We have shown that the absorption lines in the X-shooter spectrum of the afterglow of GRB 120327A are well described by a three component Voigt profile (see Table 2). Strangely enough, the three detected features are distributed across a narrow velocity interval $\left(\approx 80 \mathrm{~km} \mathrm{~s}^{-1}\right)$. Given that the host galaxy is particularly bright and rich in metal lines, we could expect a large mass, which should correspond to a large velocity dispersion.

It is interesting to note that different species show different abundance ratios among the three components. Low ionization species show absorption in all components, with the redmost component III being less populated than components I (bluest) and II, which in turn feature a similar column density. The only exception is represented by Ca II and P II which do not present absorption in component III, with the former featuring also a higher column density in component I.

On the other hand, neutral species do not show absorption in component III (with the exception of Ar I and the saturated O I), and component I has generally a column density which is higher than that of component II. This is particularly evident in Ca I and P I, where we can only set upper limits for the component II column densities. Again, Ar I does not follow this behavior, featuring a comparable absorption in the two components.

These data can be easily explained assuming that component II is closer to the GRB explosion site than component I. 
In fact, in this scenario, part of the absorbing gas in component I can survive as neutral atoms because its distance from the GRB radiation is large enough to avoid complete ionization of the atomic species. The larger column density of component I of Ca II with respect to component II is explained because this species has an ionization potential of just $11.9 \mathrm{eV}$, thus smaller than that of $\mathrm{HI}$. This means that the hydrogen is transparent to a large portion of photons able to ionize Ca II to Ca III (those with energy between 11.9 and $13.6 \mathrm{eV}$ ), and is not able to screen this transition.

Similarly, neutral species show a fainter absorption (if any) in the second component with respect to the first one due to their ionization potential being lower than that of hydrogen. Ar I is an exception, because of the comparable column densities for components I and II. This is not unexpected, since Ar I has the highest ionization potential among the neutral species, well above that of HI. This scenario is confirmed by the $\mathrm{H}_{2}$ molecule being detected in component I, and not in component II, where the GRB flux is possibly too high to allow $\mathrm{H}_{2}$ to survive.

This scenario is confirmed by the determination of the GRB/absorber distances using the excited line variation and ratios: component I is $\sim 200$ pc away from the GRB, component II $\sim 100 \mathrm{pc}$. These distances are among the shortest computed using this kind of analysis, but fully consistent with other determinations (see Hartoog et al. 2013). Moreover, the marginal or absent detection in component II of many neutral species with a low ionization potential is not unexpected. In fact, the GRB flux should fully ionize $\mathrm{Mg}$ I into $\mathrm{Mg}$ II on a distance scale of 50-100 pc (Prochaska et al. 2006).

The comparison between the Fe II excited levels and a timedependent photo-excitation code gives us another piece of information about the gas geometry around GRB 120327A. In fact, the modeling requires less ground state Fe II column densities both in component I and II than those observed, in order to reproduce the behavior of the excited levels. This is a possible indication that only a fraction of Fe II resides at $100 \mathrm{pc}$ and $200 \mathrm{pc}$ from the GRB. In other words, most of the gas detected in component I and II could be located far away from the GRB, in a region where the Fe II excited features had enough time to deexcite into the ground level when the X-shooter observation began. This statement is supported by the absorption spike present in component I of some lines, such as Si II $\lambda 1808$, Al III $\lambda 1854$ and Fe II $\lambda 2260$ (see Figs. 2 and 3), a feature that can not be closely matched by our fits performed at the nominal X-shooter resolution.

Several high ionization lines have been detected (Fig. 6, Table 2). The best fit is obtained with a single Voigt profile, centered exactly in the middle between components I and II. The only exception is represented by $\mathrm{NV}$, whose profile is nicely lined-up with component II. A more detailed modeling is not possible, since many of these lines are saturated or fall in the UVB arm, which has a lower resolution. However, the N v fit might suggest that high ionization lines tend to prefer component II, the closer to the GRB, maybe due to a strong ionization effect (see De Cia et al. 2012; Vreeswijk et al. 2013), but this is definitely not a conclusive argument.

Concerning the shape of the high ionization transitions, Fox et al. (2008) showed that, on average, they present blue wings or high velocity components with respect to low ionization species, suggesting that they may arise in a separate phase. This different morphology does not arise in the absorption features of GRB 120327A, with the possible exception of the O VI lines, which are however heavily saturated. Prochaska et al. (2008) noted that GRB sightlines show more frequent and much stronger $\mathrm{N} v$ than random sightlines through high redshift galaxies and that this, together with a line profile similar to that of fine structure lines, hints toward a circumburst origin of the $\mathrm{N} \mathrm{v}$. They concluded that if the latter is produced by photoionization of N IV by the burst radiation, it has to reside at distances on the order of $10 \mathrm{pc}$ from the GRB and show strong variability. This turned out not to be the case for GRB 080310 , for which time resolved high resolution spectroscopy was available. For this GRB, a foreground host galaxy ISM scenario is favored for the production of N V (De Cia et al. 2013). However, the N V, C IV and Si IV column densities are still way lower than what observed in X-ray spectra. The X-ray absorption is likely dominated by the circum-burst medium, where the gas is extremely ionized, much more than where N V, C IV and Si IV are (see Watson et al. 2013; Schady et al. 2011). For what concerns GRB 120327A, the $\mathrm{S} / \mathrm{N}$ of the second $\mathrm{X}$-shooter observation is not sufficient to assess the variability of $\mathrm{N} \mathrm{V}$.

\subsection{The GRB 120327A host galaxy}

Relative abundances of the GRB 120327A host galaxy have been computed for all the species detected in absorption (see Table 4). While performing this computation, we did not take high ionization lines into account. Nevertheless, the abundances computed including or not the high ions do not differ much, because high ionization column densities are much smaller than lower ionization ones for GRB 120327A (not considering the extremely saturated oxygen). The element for which this difference is higher is Phosphorus. In this case, $\log N / \mathrm{cm}^{-2}=14.19 \pm 0.04$ $(14.25 \pm 0.04)$ excluding (including) the $\mathrm{P}$ V column density.

The metallicity computed from the less refractory elements is between -1.4 and -1.1 with respect to solar values (see Table 4). This value lies in the middle of the metallicity distribution of GRB host galaxies (Savaglio 2012), which extends from $[X / H]$ less than -2 as in GRB 090926A at $z=2.11$ (Rau et al. 2012; D'Elia et al. 2012), up to super solar values as in GRB 090323 at $z=3.57$ (Savaglio et al. 2012).

Using our late time GTC photometry, the detection of the host in the $r$ band can be used to derive the star-formation rate, not corrected for host dust extinction (Savaglio et al. 2009). Using their Eq. (5), a magnitude of $r^{\prime}=24.5$, translates into a lower limit for the star-formation rate of $S F R \sim 5 M_{\odot} \mathrm{yr}^{-1}$.

Concerning the GRB 120327A host characterization, the $\alpha$ element enhancement with respect to iron group elements points toward a massive galaxy. In addition, if we correct our $r^{\prime}$ band late-time photometry for Galactic reddening, we obtain $r^{\prime} \sim 23.7$ for the host galaxy. This is quite a bright value for a GRB host at $z=2$.8. For example, GRB 021004 at $z=2.33$ has $R=24.1$ and $\log M / M_{\odot} \sim 10.2$ (Savaglio et al. 2009). Indeed, Hjorth et al. (2012) showed that none of the TOUGH hosts at $2<z<3$ is brighter than $R \sim 24$ (Vega). Finally, a comparison with chemical evolution models (Matteucci 2001; Calura et al. 2008, 2009; Grieco et al. 2012) favors a strong star formation efficiency $\left(10 \mathrm{Gyr}^{-} 1\right)$ in the GRB host, with a value similar to those typical of massive proto-spheroids (e.g., Pipino et al. 2011). It is worth to stress that single GRB-DLA studies sample only a single sightline through the host galaxy, and that different physical conditions may characterize other sightlines. However, by extending this study to a larger set of GRB hosts, in principle it could be possible to have more precise information on the global chemical abundance pattern and on the star formation history of such systems as a population. This task is deferred to future work and is already feasible, as more and more new abundances from GRB absorption studies have become available in 
the past few years. Even more will become available thanks to next generation of medium/high resolution spectrographs, such as ESPRESSO and ELT-HIRES, instruments optimized to perform absorption line studies at best and which, in principle, will offer the opportunity to probe various lines-of-sight of even distant, single objects. In this framework, the utility of chemical evolution models as unique tools to gain fundamental information on a few basic, fundamental parameters of GRB hosts such as their nature and ages, should be seriously considered.

Apparently, the aforementioned massive galaxy as the host for GRB 120327A is against the claim that most of the GRB hosts are small, star-forming, blue and metal-poor galaxies (see, e.g., Vreeswijk et al., 2001; Christensen et al. 2004; Fruchter et al. 2006; Wiersema et al. 2008; Levesque et al. 2010). However, this picture is based on samples constituted mainly by low redshift galaxies $(z<2)$. Recent, deep observations seem to suggest that this could be only a partial view, and that at high redshift the situation might be different (Krühler et al. 2011; Hunt et al. 2011; Savaglio et al. 2012; Elliot et al. 2013, Perley et al. 2013). As suggested by Savaglio (2012), the key role of this picture must be played by the star formation, which appears to be strong in most of the GRB host galaxies. GRBs are associated with the death of massive and short-lived stars $\left(M>30 M_{\odot}\right.$, see e.g. Heger et al. 2003), which in turn must reside in highly star-forming environments. In addition, star-formation in the local universe mainly occurs in small, blue galaxies, i.e., the typical GRB hosts at low $z$. On the other hand, most of the starformation at higher redshift is likely to occur in more massive galaxies (Juneau et al. 2005; Hunt et al. 2011). Thus, it is not implausible to expect that some GRB hosts at $z>2$ could be high mass galaxies, given also that many dark GRB hosts are massive (e.g. Berger et al. 2007; Levan et al. 2006; Svensson et al. 2012; Perley et al. 2013). Our result, which seems to point towards a high mass for the GRB 120327A host galaxy, confirms that the morphology of high redshift GRB hosts may not resemble that of low redshift ones.

\subsection{Dust content and molecules}

The formation of stars is tightly correlated with the presence of $\mathrm{H}_{2}$ (e.g., Shu et al. 1987; Blitz 1993; McKee \& Ostriker 2007; Bigiel et al. 2008). Thus, molecular gas is expected to be found in star-forming environments such as GRB surroundings, but the search for its absorption features has often given negative results (see e.g. Tumlinson et al. 2007). Fynbo et al. (2006) interpreted an absorption feature of the GRB 060206 afterglow spectrum as a possible $\mathrm{H}_{2}$ detection. Prochaska et al. (2009) and Sheffer et al. (2009) report the presence of strong $\mathrm{H}_{2}$ and $\mathrm{CO}$ absorption features in the spectrum of the GRB 080607 afterglow. More recently, Krühler et al. (2013) reported a strong absorption of $\mathrm{H}_{2}$ in the X-shooter spectrum of GRB 120815A, at $z=2.36$, finding a molecular fraction of $f=0.07$.

For GRB 120327A we found $f=5 \times 10^{-7}-10^{-4}$, which is the third detection of molecules in a GRB environment. The molecular features are perfectly lined up with component I. This, however, is not enough to assess that the molecules are located at a distance of $\sim 200 \mathrm{pc}$ from the GRB, because, as noted throughout the paper, both components I and II receive contribution in absorption also from gas which is far away from the GRB. The molecular fraction in GRB 120815A is more than two orders of magnitude higher than what we found for GRB 120327A, although the two GRBs feature an almost identical, large $N_{\mathrm{H}}$ column density of $\log \left(N_{\mathrm{H}} / \mathrm{cm}^{-2}\right) \sim 22$ and metallicity $([\mathrm{Zn} / \mathrm{H}] \sim$ $-1.1)$. More surprisingly $\left(\mathrm{H}_{2}\right.$ is generally stronger in dust rich environments), our burst appears not to have a high dust content. For GRB $120327 \mathrm{~A}$ we measure $[\mathrm{Zn} / \mathrm{Fe}]=0.56 \pm 0.14$, whereas, for GRB 120815A, Krühler et al. (2013) report a value of $[\mathrm{Zn} / \mathrm{Fe}]=1.01 \pm 0.10$, which means that a higher quantity of dust is present along the line of sight of this GRB. This is important, because dust plays a major role for the presence of molecular gas by catalyzing the formation of molecules on the surface of dust grains as well as shielding against Lyman-Werner photons. Guimaraes et al. (2012) studied the QSO-selected system SDSS J081634+144612 at $z=3.287$, which shows nearly identical $N_{\mathrm{HI}},[\mathrm{Zn} / \mathrm{H}]$ and $[\mathrm{Zn} / \mathrm{Fe}]\left(\log \left(N_{\mathrm{H}} / \mathrm{cm}^{-2}\right)=22.0 \pm 0.1\right.$, $[\mathrm{Zn} / \mathrm{H}]=-1.10 \pm 0.10,[\mathrm{Zn} / \mathrm{Fe}]=0.48 \pm 0.06)$. They detect an $\mathrm{H}_{2}$ molecular fraction of $f \sim 9 \times 10^{-4}$, a value closer to that of GRB 120327A. Noterdaeme et al. (2008) reported that in QSO-DLAs the Iron locked into dust $\left(N_{\mathrm{Fe}, \text { dust }}\right)$ is a primary driver of $\mathrm{H}_{2}$ detection, and that $N_{\mathrm{Fe} \text {,dust }}$ can be linked to a combination of metallicity, dust depletion and column density. Our measurement thus confirms the importance of dust in the molecular formation.

\section{Conclusions}

In this paper we present multi-epoch, intermediate-resolution $(R=7000-12000)$ spectroscopy of the optical afterglow of GRB 120327A, observed using the X-shooter spectrograph at the VLT, $\sim 2.5$ and $27.7 \mathrm{hr}$ after the trigger.

Our main findings are summarized below.

(i) From the detection of hydrogen and metal absorption features, we find that the redshift of the host galaxy is $z=$ $2.8145 \pm 0.0001$. The ISM of the GRB host galaxy has at least three components contributing to the absorption. The line center of component I (III) is 41 (35) $\mathrm{km} \mathrm{s}^{-1}$ bluewards (redwards) of component II, which sets the redshift reference point.

(ii) Component III presents less absorption features and no excited lines, and is probably the farthest from the GRB explosion site.

(iii) Components I and II have similar absorption profiles, but also some differences, with the former showing more absorption from neutral elements and the latter from highly ionized species. Fine structure and metastable levels are present in both components, but with higher column densities in component II. This points towards component II being closer to the GRB than component I.

(iv) High ionization lines do not present blue wings or higher velocity components with respect to low ionization ones, but show a similar velocity range, contrary to what is observed in previous GRBs.

(v) The distance of these components from the GRB is computed using a time dependent photo-excitation code and comparing the results with the excited line ratios for the Fe II ion. Component I is $200_{-60}^{+100}$ pc away from the GRB explosion site, while component II is at $100_{-30}^{+40} \mathrm{pc}$, confirming result (iii). The variability of the Fe II $\lambda 2396$ fine structure line between the two X-shooter spectra proves that the excited levels are produced by the GRB UV flux.

(vi) The column density of the Fe II ground state is in excess from what is expected using the photo-excitation code, both in component I and II. This possibly indicates that most of Fe II lies far away from the GRB, and that the geometry of the GRB 120327A host galaxy absorber is 
more complex with respect to the resolving capacity of $\mathrm{X}$-shooter.

(vii) The damped Lyman- $\alpha$ system associated with the GRB host galaxy is prominent, with a column density of $\log \left(N_{\mathrm{H}} / \mathrm{cm}^{-2}\right)=22.01 \pm 0.09$. The metallicity computed using the less depleted elements is between $[X / \mathrm{H}]=-1.3$ and -1.1 , i.e., a value in the middle of the metallicity distribution for the GRB host galaxies at high redshift.

(viii) The dust depletion pattern indicates an $\alpha$ element enhancement with respect to the elements of the Iron group, typical of massive galaxies. The same conclusion is supported by modeling this overabundance using detailed chemical enrichment codes.

(ix) A joint analysis of host galaxy photometry and chemical enrichment codes leads us to suggest that the host is a high mass $\left(>10^{10} M_{\odot}\right)$, high luminosity one.

(x) Concerning the dust content in the host, we estimate $A_{V}=$ $0.22 \mathrm{mag}$ along the line of sight of GRB 120327A, using the dust depletion pattern, considerably in excess of what we find studying the extinction curve shape by modeling the flux-calibrated spectra $\left(A_{V}<0.03 \mathrm{mag}\right)$. The ratio $[\mathrm{Zn} / \mathrm{Fe}]=0.56 \pm 0.14$ depicts an environment with a certain dust depletion, but not so extreme as in other dusty GRBs such as GRB 120815A or GRB 080607.

(xi) The $\mathrm{H}_{2}$ molecule is firmly detected in the GRB 120327A host galaxy. This is the third secure detection of molecules in a GRB environment after GRB 080607 and GRB 120815A. The molecular fraction we determined is at least two orders of magnitude below that of the other GRBs, possibly because of the lower dust content, since it helps the molecule formation.

Acknowledgements. We thank an anonymous referee for several helpful comments that improved the quality and clarity of the paper. We thank Eros Vanzella for several helpful discussion. V.D.E. acknowledges partial support from PRIN MIUR 2009. J.P.U.F. and D.X. acknowledge support from the ERC-StG grant EGGS-278202. A.d.U.P. acknowledges support by the European Commission under the Marie Curie Career Integration Grant programme (FP7-PEOPLE2012-CIG 322307). The research activity of A.d.U.P. and J.G. is supported by Spanish research project AYA2012-39362-C02-02. F.C. acknowledges support from INAF under the contract PRIN-INAF-2012 and from PRIN MIUR 20102011, project "The Chemical and Dynamical Evolution of the Milky Way and Local Group Galaxies", prot. 2010LY5N2T. D.M. acknowledges the Instrument Center for Danish Astrophysics for support. E.P. acknowledges INAF PRIN 2011 and ASI INAF contract I/088/06/0. The Dark Cosmology Centre is funded by the Danish National Research Foundation. The 0.82 m IAC80 Telescope is operated on the island of Tenerife by the Instituto de Astrofísica de Canarias in the Spanish Observatorio del Teide. We acknowledge Christophe Martayan (ESO) for assistence to address the malfunctioning of the X-shooter ADCs.

\section{Appendix A: IAC-80 photometry}

In this appendix we present the IAC-80 data, used to produce the lower panel of Fig. 1.

\section{Appendix B: Equivalent widths}

In this appendix we present the rest-frame equivalent widths of the most important transitions reported in Table B.1. These equivalent widths represent the sum of the contributions of all the three components which model each transition (see Sect. 4.2).
Table A.1. IAC-80 photometric I-band observations in the Vega system.

\begin{tabular}{lccc}
\hline \hline Hours from GRB & Exp. time $(\mathrm{h})$ & mag & mag error \\
\hline 1.23 & 0.08 & 17.32 & 0.05 \\
1.34 & 0.08 & 17.42 & 0.05 \\
1.44 & 0.08 & 17.50 & 0.05 \\
1.54 & 0.08 & 17.56 & 0.05 \\
1.65 & 0.08 & 17.70 & 0.05 \\
1.76 & 0.08 & 17.76 & 0.05 \\
1.86 & 0.08 & 17.81 & 0.06 \\
1.96 & 0.08 & 17.96 & 0.05 \\
2.07 & 0.08 & 17.92 & 0.05 \\
2.17 & 0.08 & 18.03 & 0.05 \\
2.28 & 0.08 & 18.12 & 0.05 \\
2.38 & 0.08 & 18.19 & 0.07 \\
2.48 & 0.08 & 18.31 & 0.08 \\
2.59 & 0.08 & 18.17 & 0.13 \\
\hline
\end{tabular}

Table B.1. Rest-frame equivalent widths (expressed in $\AA$ ) for some representative absorption features.

\begin{tabular}{lllllc}
\hline \hline Line & $E W$ & $\Delta E W$ & Line & $E W$ & $\Delta E W$ \\
\hline C II $\lambda 1334$ & 0.99 & 0.04 & C II $\lambda 1335^{*}$ & 0.51 & 0.04 \\
C IV $\lambda 1548$ & 0.75 & 0.04 & C IV $\lambda 1550$ & 0.66 & 0.04 \\
N I $\lambda 1134 \mathrm{c}$ & 0.44 & 0.06 & N V $\lambda 1238$ & 0.11 & 0.04 \\
N V $\lambda 1242$ & 0.06 & 0.04 & O I $\lambda 1302$ & 0.92 & 0.04 \\
O I $\lambda 1306 * 2$ & 0.21 & 0.04 & O VI $\lambda 1031$ & 1.37 & 0.08 \\
Mg I $\lambda 2852$ & 0.80 & 0.04 & Mg II $\lambda 2796$ & 2.07 & 0.04 \\
Mg II $\lambda 2803$ & 1.97 & 0.04 & Al II $\lambda 1670$ & 1.00 & 0.03 \\
Al III $\lambda 1854$ & 0.34 & 0.04 & Al III $\lambda 1862$ & 0.18 & 0.04 \\
Si II $\lambda 1526$ & 1.07 & 0.05 & Si II $\lambda 1808$ & 0.63 & 0.10 \\
Si II $\lambda 1309 *$ & 0.30 & 0.04 & Si IV $\lambda 1393$ & 0.48 & 0.03 \\
Si IV $\lambda 1402$ & 0.34 & 0.03 & PI $\lambda 1674$ & 0.03 & 0.02 \\
P II $\lambda 1152$ & 0.17 & 0.05 & P V $\lambda 1117$ & 0.15 & 0.08 \\
S II $\lambda 1250$ & 0.30 & 0.04 & S II $\lambda 1253$ & 0.44 & 0.04 \\
S VI $\lambda 944$ & 0.20 & 0.12 & C II $\lambda 1334$ & 0.51 & 0.04 \\
Ar I $\lambda 1066$ & 0.22 & 0.07 & Ca I $\lambda 4227$ & 0.16 & 0.06 \\
Ca II $\lambda 3934$ & 0.98 & 0.06 & Ca II $\lambda 3969$ & 0.94 & 0.06 \\
Cr II $\lambda 2056$ & 0.35 & 0.03 & Cr II $\lambda 2066$ & 0.27 & 0.03 \\
Fe II $\lambda 1608$ & 0.91 & 0.03 & Fe II $\lambda 2344$ & 1.51 & 0.22 \\
Fe II $\lambda 1618 *$ & 0.07 & 0.03 & Fe II $\lambda 2396 *$ & 0.85 & 0.22 \\
Fe II $\lambda 1629 * 2$ & 0.06 & 0.03 & Fe II $\lambda 1636 * 3$ & 0.51 & 0.04 \\
Fe II $\lambda 1642 * 3$ & 0.04 & 0.03 & C II $\lambda 1334$ & 0.51 & 0.04 \\
Fe II $\lambda 2414$ & 0.16 & 0.12 & Fe II $\lambda 1702^{* 5}$ & 0.19 & 0.03 \\
Fe II $\lambda 1712 * 6$ & 0.04 & 0.03 & Fe II $\lambda 1635^{* 9}$ & 0.04 & 0.03 \\
Ni II $\lambda 1701$ & 0.21 & 0.03 & Ni II $\lambda 1741$ & 0.25 & 0.03 \\
Ni II $\lambda 2217 * 2$ & 0.41 & 0.05 & Ni II $\lambda 2223 * 2$ & 0.15 & 0.05 \\
Zn II $\lambda 2026$ & 0.34 & 0.03 & & & \\
\hline
\end{tabular}

\section{References}

Asplund, M., Grevesse, N., Sauval, A. J., \& Scott, P. 2009, ARA\&A, 47, 481 Berger, E., Penprase, B. E., Cenko, S. B., et al. 2006, ApJ, 642, 979

Berger, E., Fox, D. B., Kulkarni, S. R., Frail, D. A., \& Djorgovski, S. G. 2007, ApJ, 660, 504

Bigiel, F., Leroy, A., Walter, F., et al. 2008, AJ, 136, 2846

Blitz, L. 1993, in Protostars and Planets III, eds. E. H. Levy, \& J. I. Lunine, 125 Bohlin, R. C., \& Gilliland, R. L. 2004, AJ, 128, 3053

Calura, F., Pipino, A., \& Matteucci, F., 2008, A\&A, 478, 335

Calura, F., Dessauges-Zavadski, M., Prochaska, J. X., \& Matteucci, F. 2009, ApJ, 639,1236

Cepa, J., Aguiar-González, M., Bland-Hawthorn, J., et al. 2003, Proc. SPIE, 4841, 1739

Christensen, L., Hjorth, J., \& Gorosabel, J. 2004, A\&A, 425, 913

Covino, S., D’Avanzo, P., Klotz, A., et al. 2008, MNRAS, 388, 347

Covino, S., Fugazza, D., \& Rossi, A. 2012, GRB Coordinater Network, Circular Service, 13128

Covino, S., Melandri, A., Salvaterra, R., et al. 2013, MNRAS, 432, 1231 
Cucchiara, A., Prochaska, J. X., Zhu, G., et al. 2013, ApJ, 773, 82 De Cia, A., Ledoux, C., Vreeswijk, P. M., et al. 2012, A\&A, 545, A64 De Cia, A., Ledoux, C., Vreeswijk, P. M., et al. 2013, A\&A, 560, A88 D'Elia, V., Fiore, F., Meurs, E. J. A., et al. 2007, A\&A 467, 629

D'Elia, V., Fiore, F., Perna, R., et al. 2009a, ApJ, 694, 332

D'Elia, V., Fiore, F., Perna, R., et al. 2009b, A\&A, 503, 437

D’Elia, V., Fynbo, J. P. U, Covino, S., et al. 2010, A\&A, 523, A36 de Ugarte Postigo, A., Goldoni, P., Thöne, C. C., et al. 2010, A\&A, 513, A42 D’Odorico, S., Dekker, H., Mazzoleni, R., et al. 2006, SPIE Conf. Ser., 6269 Dwek, E. 1998, ApJ, 501, 643

Elíasdóttir, Á., Fynbo, J. P. U., Hjorth, J., et al. 2009, ApJ, 697, 1725

Fontana, A., \& Ballester, P. 1995, The Messenger, 80, 37

Fruchter, A. S., Levan, A. J., Strolger, A. L., et al. 2006, Nature, 441, 463

Fynbo, J. P. U., Starling, R. L., Ledoux, C., et al. 2006, A\&A, 451, L47

Fynbo, J. P. U., Prochaska, J. X., Sommer-Larsen, J., Dessauges-Zavadsky, M., \& Moller, P. 2008, ApJ, 683, 321

Grieco, V., Matteucci, F., Meynet, G., et al. 2012, MNRAS, 423, 3049

Goldoni, P., Royer, F., Francois, P., et al. 2006, in SPIE Conf. Ser., 6269, 80

Gorosabel, J., Castro-Tirado, A. J., Walker, C., et al. 2012, GRB Coordinater

Network, Circular Service, 13130

Greiner, J., Bornemann, W., Clemens, C., et al. 2008, PASP, 120, 405

Guimaraes, R., Noterdaeme, P., Petitjean, P., et al. 2012, AJ, 143, 147

Hancock, P., Murphy, T., Gaensler, B., et al. 2012, GRB Coordinater Network, Circular Service, 13180

Hartoog, O. E., Wiersema, K., Vreeswijk, P. M., et al. 2013, MNRAS, 430, 2739

Hjorth, J., Malesani, D., Jakobsson, P., et al. 2012, ApJ, 756, 187

Hunt, L. K., Palazzi, E., Rossi, A., et al. 2011, ApJ, 736, 36

Kalberla, P. M. W., Burton, W. B., Hartmann, D., et al. 2005, A\&A, 440, 775

Kann, D. A., Klose, S., Zhang, B., et al. 2010, ApJ, 720, 1513

Kelson, D. D. 2003, PASP, 115, 688

Klotz, A., Gendre, B., Boer, M., \& Atteia, J. L. 2012a, GRB Coordinater Network, Circular Service, 13124

Klotz, A., Gendre, B., Boer, M., \& Atteia, J. L. 2012b, GRB Coordinater Network, Circular Service, 13132

Klotz, A., Macpherson, D., Coward D., et al. 2012c, GRB Coordinater Network, Circular Service, 13164

Kornei, K. A., Shapley, A. E., Martin, C. L., et al. 2013, ApJ, 774, 50

Krimm, H. A., et al. 2012, GRB Coordinater Network, Circular Service, 13137

Krühler, T., Küpcü Yoldas, A., Greiner, J., et al. 2008, ApJ, 685, 376

Krühler, T., Fynbo, J. P. U., Milvang-Jensen, B., et al. 2012, GRB Coordinater Network, Circular Service, 13134

Krühler, T., Ledoux, C., Fynbo, J. P. U. et al. 2013, A\&A, 557, 18

Kuin, N. P. M., \& Sbarufatti, B. 2012, GRB Coordinater Network, Circular Service, 13138

LaCluyze, A. Haislip, J., Ivarsen, K., et al. 2012, GRB Coordinater Network, Circular Service, 13127

Ledoux, C., Vreeswijk, P. M., Smette, A., et al. 2009, A\&A, 506, 661

Levan, A. J., Fruchter, A. S., Rhoads, J., et al. 2006, ApJ, 647, 471

Levesque, E. M., Kewley, L. J., Berger, E., \& Zahid, H. J. 2010, AJ, 140, 1557

Liang, S. L. \& Li, A. 2010, ApJ, 710, 1

Malesani, D., Fynbo, J. P. U., Jakobsson, P., Jessen-Hansen, J., \& Lehtinen, J. 2012, GRB Coordinater Network, Circular Service, 13152

Martin, C. L., Shapley, A. L., Coil, A. L., et al. 2012, ApJ, 760, 127

Matteucci, F. 2001, The chemical evolution of the Galaxy (Kluwer Academic Publisher)

McKee, C. F., \& Ostriker, E. C. 2007, ARA\&A, 45, 565

Mészáros, P. 2006, Rep. Prog. Phys., 69, 2259

Modigliani, A., Goldoni, P., Royer, F., et al. 2010, SPIE Conf. Ser., 7737

Noterdaeme, P., Ledoux, C., Petitjean, P., et al. 2007, A\&A, 479, 393

Oke, J. B. 1990, AJ, 99, 1621
Pei, Y. C. 1992, ApJ, 395, 130

Perley, D. A., \& Tanvir, N. R. 2012, GRB Coordinater Network, Circular Service, 13133

Perley, D. A., Levan, A. J., Tanvir, N. R., et al. 2013, ApJ 778, 128

Pipino, A., Fan, X. L., Matteucci, F., et al. 2011, A\&A, 525, A61

Piranomonte, S., Ward, P. A., Fiore, F., et al. 2008, A\&A, 492, 775

Prochaska, J. X., Chen, H. W., \& Bloom, J. S. 2006, ApJ, 648, 95

Prochaska J. X., Chen, H. W., Dessauges-Zavadsky, E., \& Bloom, J. S. 2007, ApJ, 666, 267

Prochaska J. X., Dessauges-Zavadsky M., Ramirez-Ruiz M., \& Chen, H. W. 2008, ApJ, 685, 344

Prochaska J. X., Sheffer, Y., Perley, D. A., et al. 2009, ApJ, 691, 27

Rau, A., Savaglio, S., Krühler, T., et al. 2010, ApJ, 720, 862

Sánchez-Ramirez, R., Gorosabel, J., Castro-Tirado, A. J., Rivero, M. A., \& Gómez-Velarde, G. 2012, GRB Coordinater Network, Circular Service, 13146

Savage, B. D., \& Sembach, K. R., 1996, ApJ, 470, 893

Savaglio, S. 2012, Astron. Nachr., 333, 480

Savaglio, S., Fall, S. M., \& Fiore, F. 2003, ApJ, 585, 638

Savaglio, S., Glazebrook, K., Crampton, D., et al. 2004, ApJ, 602, 51

Savaglio, S., Glazebrook, K., Le Borgne, D., et al. 2005, ApJ, 635, 260

Savaglio, S., Glazebrook, K., \& Le Borgne, D. 2009, ApJ, 691, 182

Savaglio, S., Rau, A., Greiner, J., et al. 2012, MNRAS, 420, 627

Sbarufatti, B., Barthelmy, S. D., Geherls, N., et al. 2012, GRB Coordinater Network, Circular Service, 13123

Schady, P., Page, M. J., Oates, S., et al. 2010, MNRAS, 401, 2773

Schady, P., Savaglio, S., Krühler, T., Greiner, J., \& Rau, A. 2011, A\&A, 525, 113

Sheffer, Y., Prochaska, J. X., Draine, B. T., Perley, D. A., \& Bloom, J. S. 2009, ApJ, 701, L63

Shu, F. H., Adams, F. C., \& Lizano, S. 1987, ARA\&A, 25, 23

Smith, R. J., \& Virgili, F. 2012, GRB Coordinater Network, Circular Service, 13125

Smith, J. A., Tucker, D. L., Kent, S., et al. 2002, AJ, 123, 2121

Sparre, M, Hartoog, O. E., Krühler, T. et al. 2013, ApJ, submitted [arXiv: 1309.2940]

Srianand, R, Petitjean, P., Ledoux, C., Ferland, G., \& Shaw, G. 2005, MNRAS, 362,549

Steidel, C. C., Adelberger, K. L., Giavalisco, M., Dickinson, M., \& Pettini, M. 1999, ApJ, 519, 1

Stratta, G., Maiolino, R., Fiore, F., \& D’Elia, V. 2007, ApJ, 661, 9

Stroh, M. C., Burrows, D. N., Page, K. L., et al. 2012, GRB Coordinater Network, Circular Service, 13141

Sudilovsky, V., Nicuesa Guelbenzu, A., \& Greiner, J. 2012, GRB Coordinater Network, Circular Service, 13129

Svensson, K. M., Levan, A. J., Tanvir, N. R., et al. 2012, MNRAS, 421, 25

Tumlinson, J., Prochaska, J. X., Chen, H.-W., Dessauges-Zavadsky, M., \& Bloom, J. S. 2007, ApJ, 668, 667

van Dokkum, P. G. 2001, PASP, 113, 1420

Vernet, J., Dekker, H., D'Odorico, S., et al. 2011, A\&A, 536

Vreeswijk, P. M., Fruchter, A., Kaper, L., et al. 2001, ApJ, 546, 672

Vreeswijk, P. M., Ellison, S. L., Ledoux, C., et al. 2004, A\&A, 419, 927

Vreeswijk, P. M., Ledoux, C., Smette, A., et al. 2007, A\&A, 468, 83

Vreeswijk, P. M., Ledoux, C., Smette, A., et al. 2011, A\&A, 468, A83

Vreeswijk, P. M., Ledoux, C., Raassen, A. J. J., et al. 2013, A\&A, 532, A3

Watson, D. J., Fynbo, J. P. U., Ledoux, C., et al. 2006, ApJ, 652, 1011

Watson, D. J., Zafar, T., Andersen, A. C., et al. 2013, ApJ, 768, 23

Wiersema, K., Savaglio, S., Wreeswijk, P. M., et al. 2007, A\&A, 464, 529

Zafar, T., \& Watson, D. J. 2013, A\&A, 560, A26

Zafar, T., Watson, D., Fynbo, J. P. U., et al. 2011, A\&A, 532, A143

Zafar, T., Watson, D., Elíasdóttir, Á., et al. 2012, ApJ, 753, 82 